\title{
Two responses of a uniformly stratified flow over a sloping bottom to a spatially varying downwelling favorable wind.
}

\author{
by \\ Evgeny Logvinov \\ B.Sc. in Applied Physics and Mathematics \\ Moscow Institute of Physics and Technology, 2005 \\ Submitted in partial fulfillment of the requirements for the degree of \\ Master of Science \\ at the \\ MASSACHUSETTS INSTITUTE OF TECHNOLOGY \\ and the \\ WOODS HOLE OCEANOGRAPHIC INSTITUTION \\ September 2008 \\ (C) Evgeny Logvinov 2008. All rights reserved. \\ The author hereby grants to MIT and to WHOI \\ permission to reproduce and to distribute publicly \\ paper and electronic copies of this thesis document \\ in whole or in part.
}

Signature of Author

Joint Program in Physical Oceanography Massachusetts Institute of Technology Woods Hole Oceanographic Institution July 28, 2008

Certified by

Joseph Pedlosky

Scientist Emeritus

Thesis Supervisor

Accepted by....

Raffaele Ferrari

Chairman, Joint Committee for Physical Oceanography Massachusetts Institute of Technology Woods Hole Oceanographic Institution 


\title{
Two responses of a uniformly stratified flow over a sloping bottom to a spatially varying downwelling favorable wind.
}

by

\author{
Evgeny Logvinov
}

\author{
Submitted to the Massachusetts Institute of \\ Technology and the Woods Hole Oceanographic \\ Institution in a partial fulfillment of the requirements \\ for the degree of Master of Science
}

\begin{abstract}
The work was motivated by studies of Austin and Lentz (2002) and Pedlosky (2007). The above mentioned works considered two different responses of the stratified flow to a downwelling favorable wind forcing. The first study investigated a time dependent flow with a formation of a constantly expanding relatively well mixed region near the shore and the second considered a steady flow that arises when an offshore varying wind is applied. In my thesis I use ROMS to determine which type of response will take place based on the wind amplitude near the coast. It was demonstrated that if the value of the wind is much smaller than the critical value (determined by the stratification, the rotation rate and the horizontal diffusivity) then the flow is steady (the bbl case) and similar to the one investigated by Pedlosky. If the wind is of the order, or larger than, the critical value then the response is time dependent (the pool case) and similar to the one described by Austin and Lentz. The resulting flow structure of each response was also investigated. I examined the sensitivity of the bbl response to variations in the background vertical diffusivity, the initial stratification and the bottom slope. It was shown that a higher background vertical diffusivity, a higher stratification and a shallower bottom slope correspond to thinner (vertically) and narrower (horizontally) bbl. For the pool case the time dependent structure was also examined, using a number of idealized models. It was shown that the rate of the pool region expansion is a complex function of the local wind stress amplitude and the local depth.
\end{abstract}

Thesis Supervisor: Joseph Pedlosky

Title: Scientist Emeritus 


\section{Acknowledgments}

This work would not be possible without support from many people. First, I would like thank my advisor, Joe Pedlosky. I learned a lot from him. Most importantly, I learnt what it is like to be a scientist. Also, I learned not to be scared of page-long equations.

I am very grateful to my thesis committee members: Steve Lentz, Ken Brink and Glenn Flierl. I would like to especially thank Steve for his constant willingness to help and for his insights into coastal oceanography. A big thanks to Ken for the time he spent talking with me about numerical modeling.

I am really thankful to Andrey Shcherbina, who introduced me to the Regional Oceanic Modeling System (ROMS) and helped me to make the first and most difficult steps in understanding this numerical model. I would like to give a special thanks to John Warner as well. He spent a lot of his valuable time helping me when I had trouble with ROMS.

I am really thankful to my girlfriend and friend, Christie, for her support throughout my whole thesis preparation period and especially the last few crazy days. She was also the first reader of my draft and the person who made it understandable for the rest of humankind.

I have really big thanks to all my friends, those I already had before I entered the program and those I met during it. Special thanks to my classmates Peter, Jinbo and Christie (again!). They made these hard years of classes and thesis preparation fun and enjoyable. I am very grateful to Max and Tatiana, who were my first friends and mentors on this side of the world. I want to give a huge thank to all of the joint program students, especially those in PO. Special thanks to Yohai for being an incredible mentor and to Matt for being himself. I'd also like to thank Bea, Katie, Ariane, Melanie, Dave, Jessica, Kjetil, Hristina, Gregg, Sophie, Shaoyu, Stephanie, Carlos, for being great friends and elder PO brothers and sisters.

I owe a large debt to Andrew, who was the meanest, but best critic of my thesis draft.

Also thanks goes to Martha, Fanny, Daniel and Ian for being great officemates.

Special thanks to crEAPS, the MIT dance troupe and the MIT PE department for keeping me sane while at MIT.

Tremendous thanks goes to my family in Russia, who gave me constant moral support throughout all of my life. Without them none of this would have been possible. 


\section{Contents}

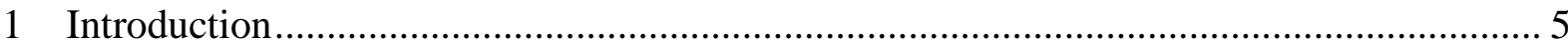

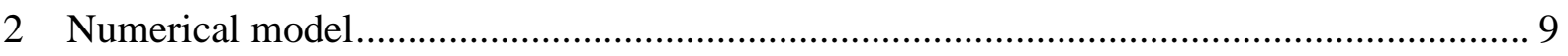

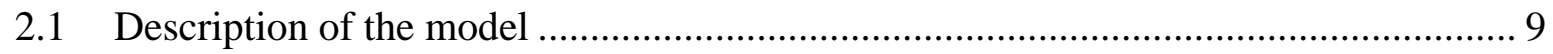

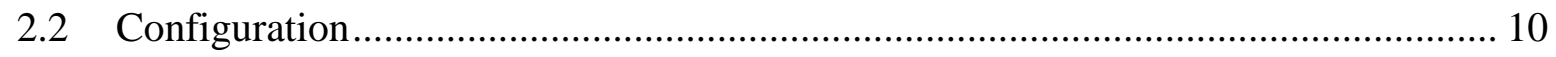

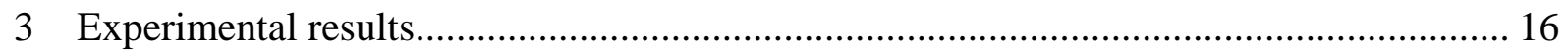

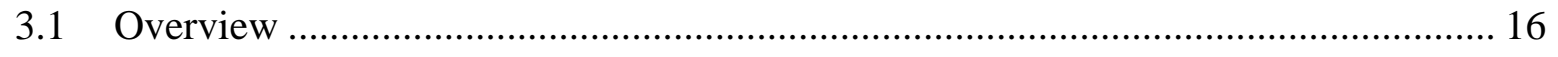

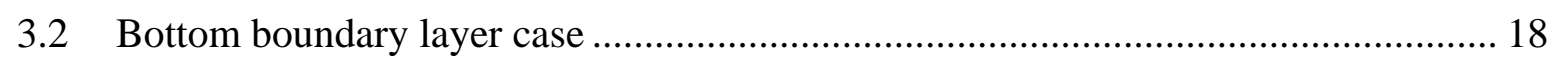

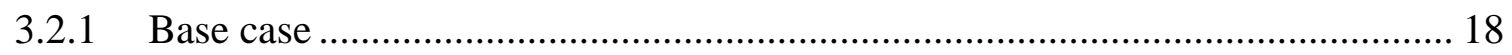

3.2.2 Experiments with one changing parameter …………................................... 27

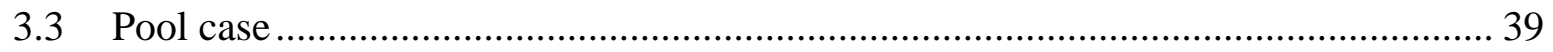

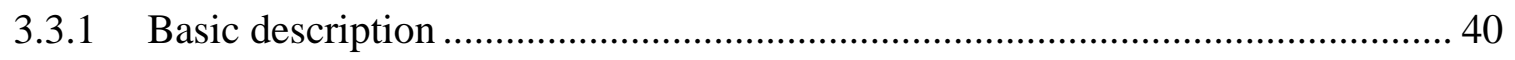

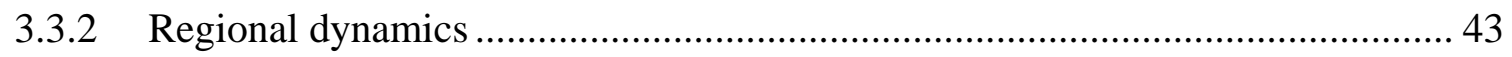

3.3.3 Temporal development............................................................................. 48

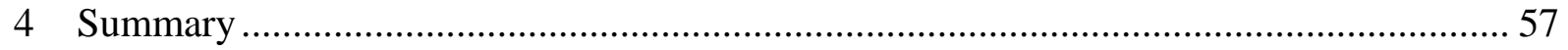

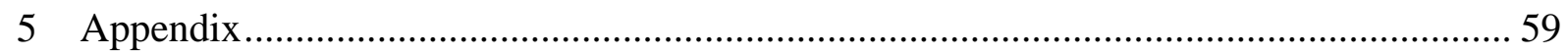

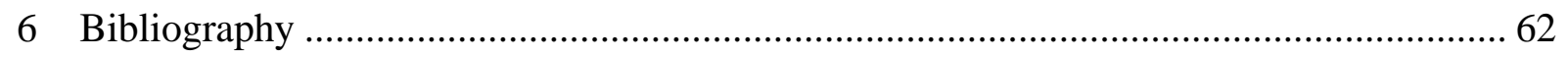




\section{Introduction}

The continental shelf is the central stage for many important oceanic processes and problems such as ocean productivity, red tides, pollution problems and water mass formation. It is no wonder that this region is the target for intense interdisciplinary research. The regional dynamics are extremely complicated and feature a large variety of forcing mechanisms such as fresh water fluxes, tides and wind. In addition, the shallow depth makes the bottom friction important and causes the flow to quickly respond to temporal changes in forcing mechanisms.

The purpose of this thesis is to investigate the dynamical fields' response to the wind forcing. Wind-driven currents are of great importance in coastal oceanography. Upwelling and downwelling flows are particularly interesting and important cases. The cross-shelf circulation associated with both types of flows is vitally important to the redistribution of tracer fields. In particular, upwelling is the dominant mechanism of enriching the surface waters with nutrients.

A wind driven, stratified, rotating flow over a flat bottom has been widely studied. In this case, the interior flow is vertically sheared and sandwiched between the surface and bottom Ekman layers (Barcelon, V. and J. Pedlosky, 1967). The presence of a sloping rigid boundary renders the problem significantly more complicated. Beginning with the work of MacCready and Rhines (1993) and Garrett et al. (1993) it became clear that the dynamics of the flow outside of the boundary layer could not be separated from the bottom boundary layer's dynamics and thermodynamics. In the sloping bottom case, Ekman transport (Ekman, 1905) causes density to be advected across the slope. In the case of upwelling, the imbalance between the pressure gradient and the Coriolis force drives fluid cross-slope in the bottom Ekman layer and the advection of density gives rise to buoyancy forces which oppose further motion. If the final 
balance between the buoyancy, pressure and Coriolis forces is achieved, there is no further Ekman flux, no fluid injected into, or sucked from, the interior, and no further spin-down of the interior flow. In such circumstances the interior sees a nearly free-slip boundary condition at the seafloor (Garrett, 1993). In the downwelling case, light water is advected beneath heavier fluid, giving rise to gravitational instability and enhanced mixing, which makes the density in the boundary layer a function of offshore position only, and thickens the bottom boundary layer (bbl). As the boundary layer thickens, the resulting horizontal density gradients reduce the bottom velocity, which in turn, decreases the transport in the bbl, making the bottom slippery ( Chapman,D.C. and S.J. Lentz, 1997).

The general response to the wind forcing was studied by Allen and Newberger (1997). Using the Princeton Ocean Model (POM) they investigated the shelf response to a downwelling favorable wind which is constant in time and uniform in space. The dominant feature of the response flow field was a downwelling front which moves offshore, leaving behind an inshore region where the density is relatively well mixed. Austin and Lentz (2002) studied both upwelling and downwelling responses of the shelf with a strong pycnocline and constant bottom slope using POM. Their work revealed significantly different responses in the two cases both in the density and velocity fields. They also reported the presence of offshore propagating downwelling/upwelling fronts and demonstrated the displacement of the front to be proportional in the former case to the square root of time and in the latter case to time.

The studies above mentioned investigated the coastal flow response to a spatially uniform wind stress. This is hardly the case in the real ocean. Lopez-Mariscal and Clarcke (1996) pointed out the importance of the offshore variation in the wind stress, referring to the observational 
wind data obtained during the Coastal Ocean Dynamics Experiment in 1981and 1982. Using a theoretical model they demonstrated that flow forced by the alongshore wind with offshore varying amplitude features two types of shear. First, the flow has a horizontal shear associated with the variation in the amplitude of the applied stress. Second, it has vertical shear associated with the second offshore derivative of the applied wind stress, in other words, with offshore variation of the Ekman pumping (e.g., Pedlosky, 1987).

The problem of the flow forced by offshore varying wind stress above a sloping bottom was further investigated by Pedlosky (2007). Using the simple theoretical model of Chapman and Lentz (1997, CL model), he obtained the expression for the bbl thickness as a function of several parameters: strength of the applied wind stress, vertical and horizontal diffusion coefficients in the interior and the horizontal diffusion in the bbl. It was also pointed out that for too large interior diffusion coefficients the bbl occupies only a finite portion of the slope.

In my thesis I am taking further steps in investigating the flows forced by spatially varying wind stress over a sloping bottom. In particular, I focus on the dynamics of the bbl. I approach the problem by examining the equilibrium state of a current driven at its upper surface by a wind stress that is variable only in the offshore direction. In the presence of a vertical density gradient driven by surface heating, the onshore flow in the upper Ekman layer is generally divergent and produces a vertical velocity in the interior and consequently a horizontal density gradient, as well as an offshore flow in the bbl. The flow in the bbl is driven by both the applied stress and by the thermal condition of no vertical density flux through the bottom. The motivation for considering a two-dimensional problem (which does not include the along-shore variation) is to examine the final equilibrium structure following Chapman and Lentz (1997) and to further enrich the 
physical context of the problem by investigating the importance of the surface forcing by the applied wind stress and dissipation in the interior.

For this study, I use the Regional Ocean Modeling System (ROMS, Shchepetkin and McWilliams, 2005) numerical model. There are a number of advantages to using numerical models to investigate this kind of problems. They have high space and time resolution, they make it possible to remove some of the constraints that make an analytical approach difficult if not impossible and they allow us to investigate a wide parameter space.

The Thesis proceeds as follows. Chapter 2 describes the model. Chapter 3 describes and interprets the results of the conducted experiments. Chapter 4 summarizes the results. 


\section{Numerical model}

The use of a numerical model allows the investigation of an idealized two-dimensional stratified coastal circulation under a wide range of conditions. In this chapter I discuss the model, the geometry of the domain, the initial stratification and the wind forcing. The chapter proceeds as follows: a description of numerical model, followed by a description of the physical parameters used in the model, finally a discussion of the numerical values of the physical parameters used in the experiments.

\subsection{Description of the model}

The Regional Ocean Modeling System (ROMS) (Shchepetkin and McWilliams, 2005) is a hydrostatic, split-explicit, primitive equation, topography-following coordinate, free-surface, incompressible numerical model written to study regional circulation. This particular numerical model has certain advantages over other models. One of the most important, for the current study, is a topography-following coordinate. This yields a smooth representation of the topography and the simulation of interactions between flows and topography, which is crucial for investigating boundary layer dynamics. In contrast, z-level models have difficulties in simulating these kinds of processes, because of a step-like representation of topography. In addition ROMS uses a split-explicit time stepping algorithm, which is more computationally cost efficient. There are other advantages that reduce the numerical error (Ezer et al., 2002). 


\subsection{Configuration}

In this study, we use a two-dimensional channel configuration eliminating alongshore variability $(x)$, leaving variation only in the vertical and cross-shelf directions $(z, y)$.

\section{Field equations}

2.1

$$
\frac{\partial u}{\partial t}+v \frac{\partial u}{\partial y}+w \frac{\partial u}{\partial z}-f v=\frac{\partial}{\partial y}\left(A_{H} \frac{\partial u}{\partial y}\right)+\frac{\partial}{\partial z}\left(A_{V} \frac{\partial u}{\partial z}\right)
$$

2.2

$$
\frac{\partial v}{\partial t}+v \frac{\partial v}{\partial y}+w \frac{\partial v}{\partial z}+f u=-\frac{1}{\rho_{0}} \frac{\partial p}{\partial y}+\frac{\partial}{\partial y}\left(A_{H} \frac{\partial u}{\partial y}\right)+\frac{\partial}{\partial z}\left(A_{V} \frac{\partial u}{\partial z}\right)
$$

2.3

$$
p_{z}=-\rho g
$$

2.4

$$
\frac{\partial \rho}{\partial t}+v \frac{\partial \rho}{\partial y}+w \frac{\partial \rho}{\partial z}=\frac{\partial}{\partial y}\left(K_{H} \frac{\partial \rho}{\partial y}\right)+\frac{\partial}{\partial z}\left(K_{V} \frac{\partial \rho}{\partial z}\right)
$$

2.5

$$
\frac{\partial v}{\partial y}+\frac{\partial w}{\partial z}=0
$$

Where $(u, v)$ are the horizontal velocity components in the $(x, y)$ direction, $\mathrm{w}$ is the vertical velocity in the $z$ direction, $\rho_{0}$ is a constant reference density, $f$ is the Coriolis parameter, $g$ is the gravity acceleration, $A_{H}$ is the constant horizontal kinematic eddy viscosity and $K_{H}\left(=A_{H}=4 \times\right.$ $10^{-2} \mathrm{~m}^{2} \mathrm{~s}^{-1}$ ) is a constant horizontal heat diffusivity. The vertical kinematic viscosity $A_{V}$ and diffusivity $K_{V}$ are given by

$$
\begin{aligned}
& A_{V}=\hat{A}_{V}+A_{V_{b a k}} \\
& K_{V}=\widehat{K}_{V}+K_{V_{b a k}}
\end{aligned}
$$


Values of $\hat{A}_{V}$ and $\widehat{K}_{V}$ are provided by the Mellor-Yamada level 2.5 turbulence closer scheme (Mellor and Yamada, 1982). Background vertical eddy viscosity and diffusivity are $A_{V_{b a k}}=$ $1.6 \times 10^{-3} \mathrm{~m}^{2} \mathrm{~s}^{-1}$ and $K_{V_{b a k}}$ respectively. The model domain is uniformly rotating $(f=$ $10^{-2} s^{-1}$ ). This high value of the Coriolis parameter is chosen in order to reduce the spin up time and to maintain thickness of the boundary layers within the appropriate limits. It corresponds to relatively small values of the deformation radius and slope Burger number (Table 2.1).

\section{Geometry}

The experimental domain is a channel uniform in along-shore direction $(x)$ with periodic boundary conditions in the momentum and tracer fields on both ends of the channel.

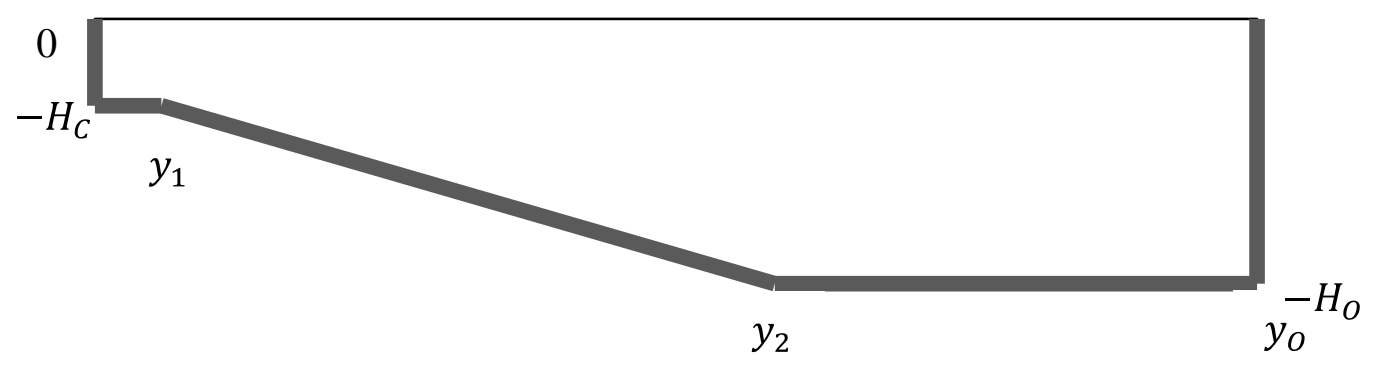

Figure 2.1. Cross-shore section of the numerical model domain.

The domain has a width of $20 \mathrm{~km}$ and depth varying cross-shore from $H_{C}$ near the coast to $H_{O}$ offshore (Figure 2.1). The lateral boundaries are vertical walls, with no inflow, no slip and insulated conditions applied. I use a wall at the coast to make sure that the vertical grid spacing will not go to zero at $\mathrm{y}=0$. I use a wall condition at the offshore side of the domain instead of open boundary condition, because of the open boundary condition complexity and inaccuracy. 
The presence of the offshore wall does not affect the dynamics of the region we are interested in. I use an idealized bathymetry consisting of three parts: a flat horizontal part adjacent to the shore at depth $H_{C}\left(y_{1}=65 \mathrm{~m}\right)$, a sloping part (depth increasing offshore, $y_{2}=12 \mathrm{~km}$ ) and a flat bottom offshore at depth $H_{O}\left(y_{O}=20 \mathrm{~km}\right)$ with smooth transitions between them. The bottom boundary conditions are that there is no vertical heat flux, no normal flow and there is a linear drag condition for momentum:

2.6

$$
\overrightarrow{\boldsymbol{\tau}}_{\text {bottom }}=\boldsymbol{r} \boldsymbol{\rho} \overrightarrow{\boldsymbol{u}}
$$

I use $r=5 \times 10^{-2} \mathrm{~m} / \mathrm{s}$. I chose this value in order to keep velocity small near the bottom.

\section{Grid}

ROMS uses the topography-following $\sigma$-coordinate. For the experiments I use the grid that has 32 vertical levels with logarithmic spacing, providing high resolution near the top and bottom with the smallest distance between the levels $0.06 \mathrm{~m}$ and the maximum $6 \mathrm{~m}$ (within the area I focus on). The horizontal spatial varies from $5 \mathrm{~m}$ near the wall to $450 \mathrm{~m}$ in the interior (Figure 2.2). Its size is determined by the criterion that the adjacent cell volume should not differ more than $10 \%$ to provide smooth numerical calculation. 


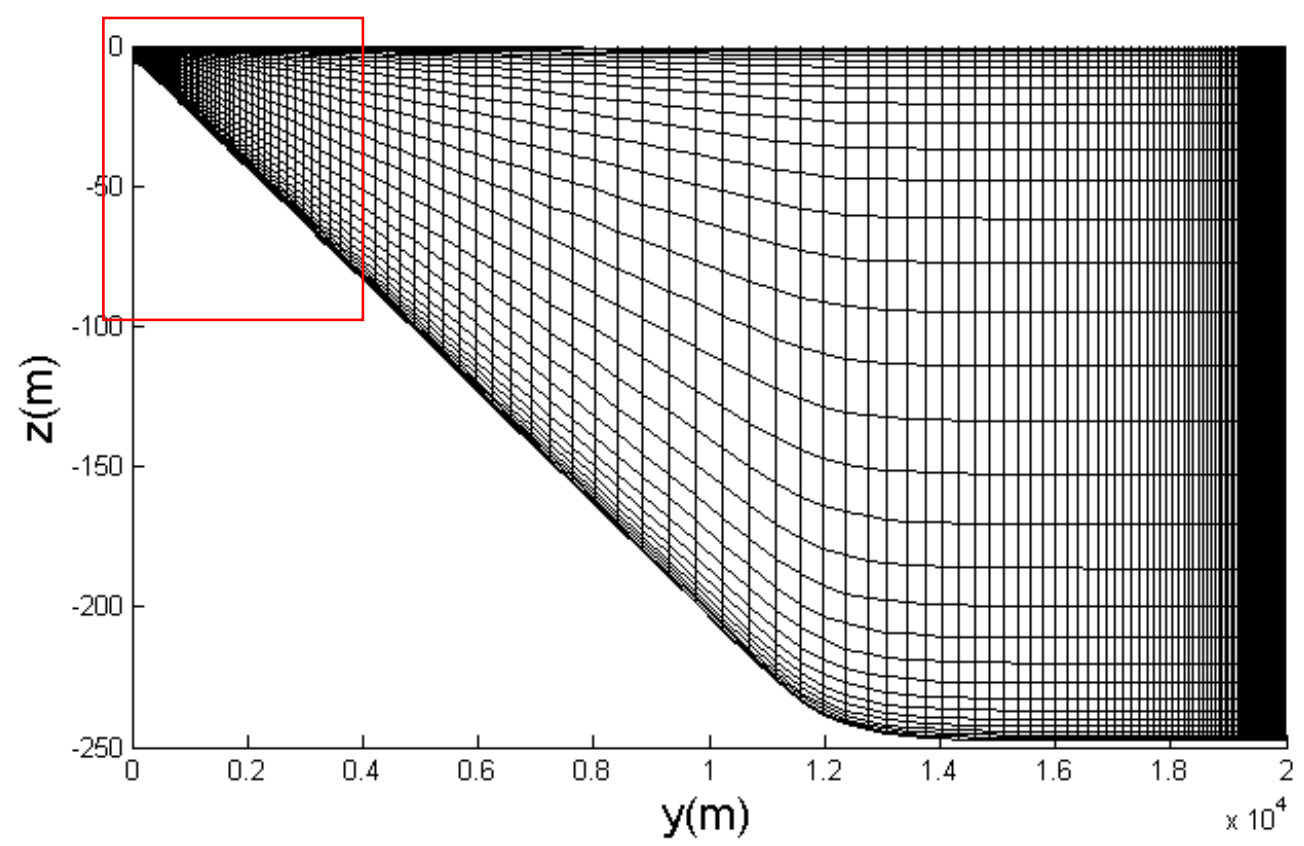

Figure 2.2. Model grid. The area I focus on is within red rectangular. The black regions near the borders correspond to the areas with very high resolution.

\section{Initialization and forcing}

Each run starts from rest. The fluid has an initial uniform stable stratification of $T_{z_{0}}$.

The flow is forced by a wind stress that is constant in time and varies offshore with only an along shore component (2.7), applied at $t=0$.

2.7

$$
\tau=\tau_{0} * \exp \left(-y / L_{w}\right)
$$

This particular structure of the wind stress was chosen for several reasons. First, it provides a smooth transition from a strong wind near the shore wall to a negligible wind stress far from the coast. This is especially important because the experimental domain has a wall on the offshore side and in the case of a significant wind stress near the offshore edge, upwelling would take place, causing disturbance in the ambient stratification. Second, an exponential form has a 
distinctive special scale $L_{w}$ that makes analysis of the results more clear. Third, this is the forcing that was used in a previous theoretical study (Pedlosky 2007) and I will compare the present study results to the results obtained in that work.

A uniform heat flux is imposed at the surface of the fluid of value $Q$ to maintain the initial stratification. This and the fact that the domain is closed introduce a slow drift to the system, but on the time scales we are interested in this drift is not important.

\section{Parameter choice}

The adjustment of the flow depends primarily on the buoyancy frequency $N$, the bottom slope $\alpha$, the wind stress amplitude $\tau_{0}$ and the characteristic wind scale $L_{w}$. The range of choices for the parameters is restricted by both the domain size and the computational cost. I use the ROMS to simulate two different responses of the flow to a downwelling wind. The parameter choices for each experiment are slightly different.

First, I discuss the parameter choices for the bottom boundary layer case (see Chapter 3 for details). After numerous trials, the most appropriate model configuration seemed to be to fix the horizontal wind scale at $L_{w}=1000 \mathrm{~m}$ and the wind amplitude at $\tau_{0}=5 * 10^{-3} \mathrm{~m}^{2} \mathrm{~s}^{-2}$ and vary the other parameters. I use one of the experiments as a base to compare with the other experiments. I call this experiment the "base case" (bc). In this case the bottom slope is $\alpha=2 *$ $10^{-2}$, the background vertical diffusivity is $K_{V_{b a k}}=10^{-6} \mathrm{~m}^{2} \mathrm{~s}^{-1}$, the initial stratification is

$T_{z 0}=0.1^{\circ} \mathrm{C} \mathrm{m}-1$ and the surface heat flux is $\frac{Q}{\rho C_{p}}=10^{-7}{ }^{\circ} \mathrm{C}^{-1}$. All other experiments are the same as the bc experiment except for a change in a one particular parameter: 
Table 2.1. Summary of the numerical experiments denoted by a concise description of the difference from the base case and the abbreviation used to designate the experiments in the text and the figures. The third column is the slope Burger number and the forth column is the Rossby deformation radius.

\begin{tabular}{|c|c|c|c|}
\hline Abbreviation & Description & $N \alpha / f$ & $N D / f$ \\
\hline $\mathrm{bc}$ & Base case & 0.026 & $6.5-120 \mathrm{~m}$ \\
\hline $2 K_{V_{b c}}$ & $K_{V_{b a k}}=2 * 10^{-6} \mathrm{~m}^{2} \mathrm{~s}^{-1}$ & 0.026 & $6.5-120 \mathrm{~m}$ \\
\hline $10 K_{V_{b c}}$ & $K_{V_{b a k}}=10^{-5} \mathrm{~m}^{2} \mathrm{~s}^{-1}$ & 0.026 & $6.5-120 \mathrm{~m}$ \\
\hline $2 N_{b c}^{2}$ & $T_{z 0}=0.2^{\circ} \mathrm{C}^{-1}$ & 0.037 & $9.1-170 \mathrm{~m}$ \\
\hline$\alpha_{b c} / 2$ & $\alpha=10^{-2}$ & 0.013 & $6.5-63.3 \mathrm{~m}$ \\
\hline$\alpha_{b c} / 8.3$ & $\alpha=2.4 * 10^{-2}$ & 0.004 & $20.4-42 \mathrm{~m}$ \\
\hline pool & Pool case & 0.005 & \\
\hline
\end{tabular}

For the other response, several experiments were conducted here I present the results from only one of them. I call this experiment the "pool" case. For this experiment the horizontal wind scale is $L_{w}=2000 \mathrm{~m}$, the wind amplitude is $\tau_{0}=0.1 \mathrm{~m}^{2} \mathrm{~s}^{-2}$, the slope is $\alpha=1.2 * 10^{-3}$, the background diffusivity is $K_{V_{b a k}}=10^{-6} \mathrm{~m}^{2} \mathrm{~s}^{-1}$, the initial stratification is $T_{z 0}=1{ }^{\circ} \mathrm{C} \mathrm{m} \mathrm{m}^{-1}$ and the surface heat flux is $\frac{Q}{\rho C_{p}}=10^{-7}{ }^{\circ} \mathrm{C} \mathrm{ms}^{-1}$.

Experimental results are presented in the next chapter. 


\section{Experimental results}

\subsection{Overview}

In this chapter, the results from the numerical experiments will be shown and discussed. The numerical experiments (Table 2.1) revealed two distinctive cases of downwelling response.

The first kind of response is observed in cases where the applied wind is weak. In this case I observe a vertically layered flow structure (Figure 3.1, A), consisting of the surface Ekman layer, an unstratified bottom boundary layer (bbl) and a stratified interior. In this case flow reaches a quasi equilibrium state after a certain period of time (depending on the stratification, the bottom slope, the background vertical diffusivity). I will refer to this case as bottom boundary layer case.

The second kind of response takes place when a strong wind is applied. The criterion, that determines if the wind is strong, is that the applied wind amplitude (wind stress at the coastal wall, $\mathrm{y}=0$ ) is larger or of the order of $\tau_{c}$ (see Appendix):

$$
\tau_{c}=\frac{K_{H} \rho_{0} f^{2}}{\sqrt{\kappa g T_{z}}}
$$

Where $\kappa$ is the thermal expansion coefficient; $T_{z}$ is the initial vertical temperature gradient; $g$ is the gravitational constant; $f$ is the Coriolis parameter; $K_{H}$ is the horizontal diffusivity; $\rho_{0}$ is the reference density. 


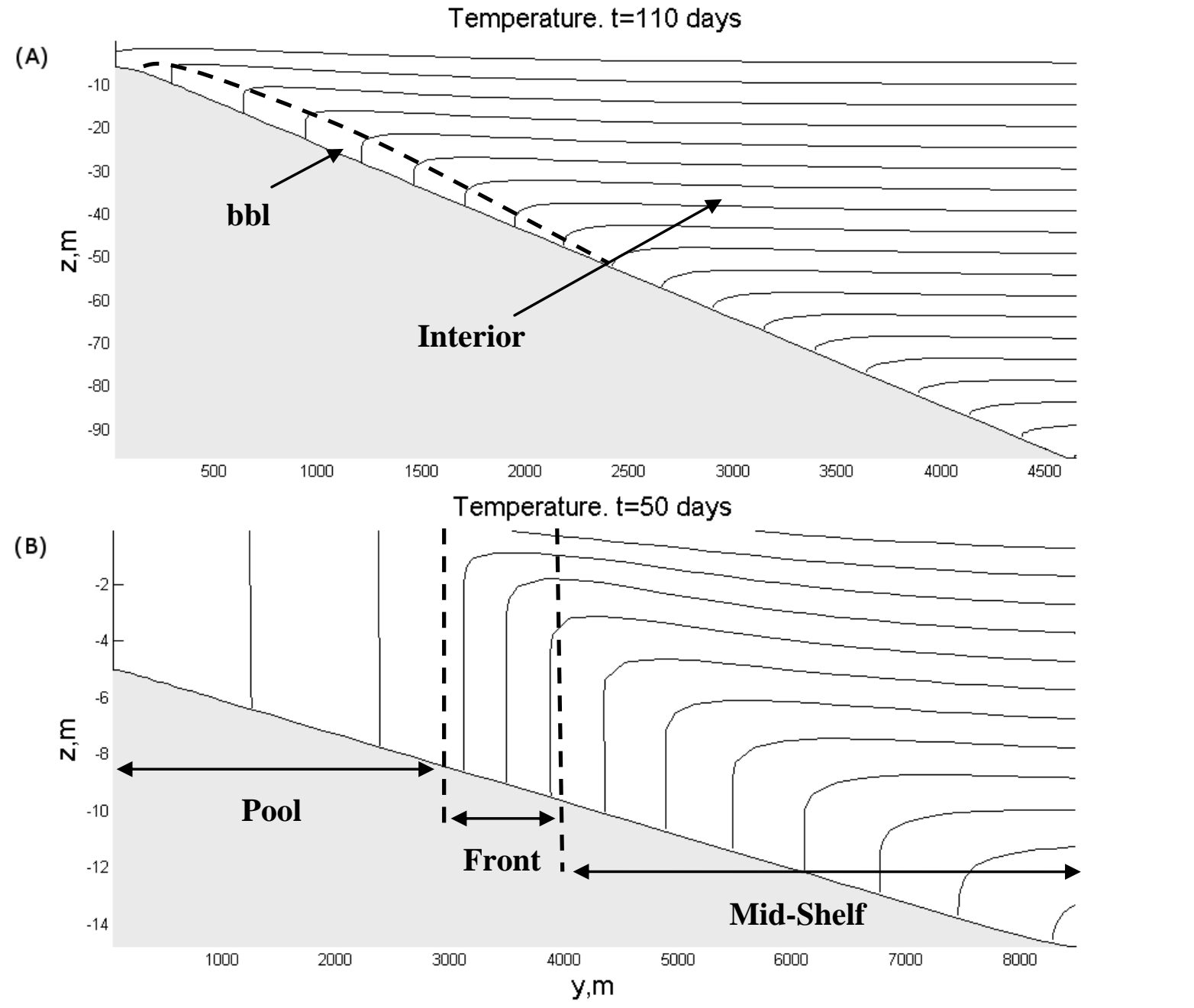

Figure 3.1. Two different cases of the downwelling response. Temperature contours in the cross-shore section. The results shown are from experiments: (A) bc after 110 days of run and (B) pool after 50 days of run. In the bc case flow reaches quasi equilibrium state. In the pool case flow did not reached a steady state at all and presented distribution is a snap shot. The contour steps are $0.5^{\circ} C$ in the be case and $1^{\circ} C$ in the pool case. Dashed lines separate the zones of different dynamics.

In this case, a region of vertically well-mixed water is formed near the coastal boundary (Figure 3.1, B). I call this region the "pool". On the offshore side of the pool a strong barotropic offshore temperature gradient is present. I refer to this region as the "front". Offshore of the front the flow has a structure similar to the bbl case. I call this region the "mid-shelf". The flow in this 
case does not reach a steady state and evolves with time. I will refer to this case as the "pool case".

This chapter proceeds as follows. Section 3.2 describes the adjustment of the bottom boundary layer case and its dynamics in the final state after 110 days based on the results from bc experiment (Subsection 3.2.1). It also discusses the dependence of the bbl case response on the background diffusivity, the initial stratification and the bottom slope based on the results from the experiments where only one of these parameters was varied (Subsection 3.2.2).

Section 3.3 deals with pool case dynamics and temporal development.

\subsection{Bottom boundary layer case}

This section discusses the results from a number of numerical experiments (Table 2.1), in which bbl case response was observed. Several experiments were conducted in which only one parameter was altered to investigate the dependence of the response on the background vertical diffusivity, background stratification and bottom slope.

\subsubsection{Base case}

The flow in the base case represents a common behavior of the bbl case response. Here, using the results from the bc experiment, I will discuss the adjustment and state of the flow after 110 days of run. 


\subsubsection{Adjustment}

After a time period of the order of the spin up time ( $\sim 0.7$ days $)$, the alongshore velocity and cross-shore streamfunction have structures similar to what we would expect in the homogenous case (Figure 3.2, A). The geostrophicaly balanced interior is sandwiched between the surface and the bottom Ekman layers. In the interior, the offshore decaying alongshore velocity is almost barotropic and has a magnitude of the order (e.g., Pedlosky, 1987):

$$
U(y)=\frac{2 \tau(y)}{\rho f \delta_{E}}
$$

As time goes by, the offshore flow in the bottom Ekman layer advects lighter water beneath heavier fluid, which leads to static instability, causing intense vertical mixing. This mixing alters the temperature field such that a bottom boundary layer with vertically well mixed temperature is formed (Figure 3.2, B, C). Here I define the bbl as an area near the bottom where the stratification is less than $0.02 \%$ of the initial stratification. The interior has a stable stratification and, because the isopycnals are continuous through the top of the bbl (top of the bbl has a negative slope), a negative offshore temperature gradient is formed in the bbl. In the bbl, the alongshore velocity is in geostrophic balance (see next subsection) with the offshore temperature gradient and according to the thermal wind relationship has a positive vertical shear. With time, the bbl thickens (Figure 3.3) and the alongshore velocity accelerates (Figure 3.4). 
(A)

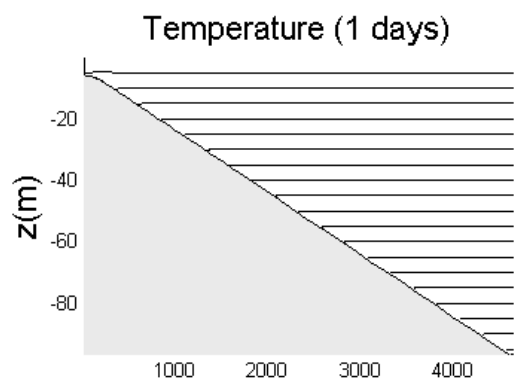

(B)

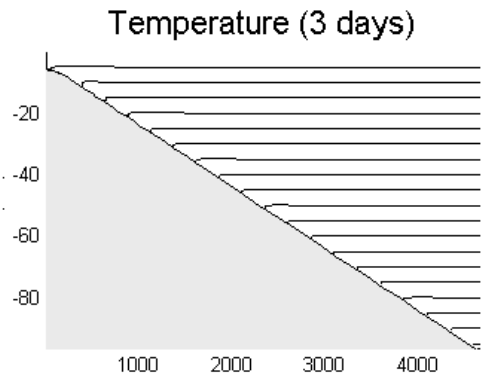

(C)

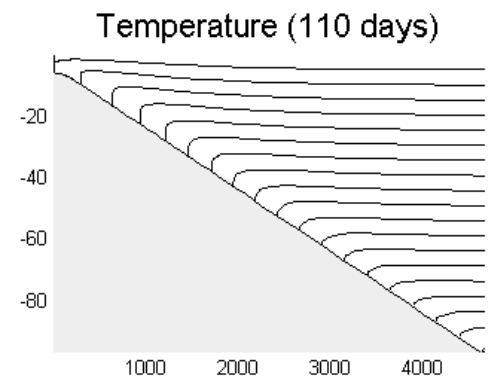

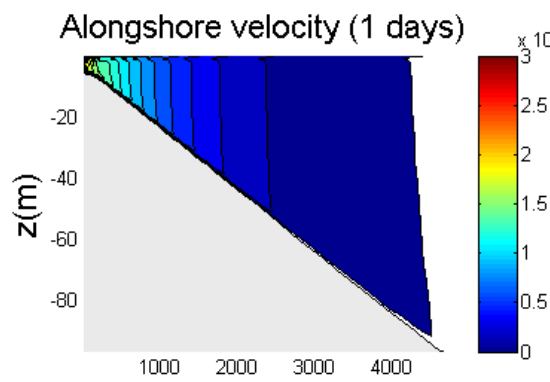

Alongshore velocity ( 3 days)
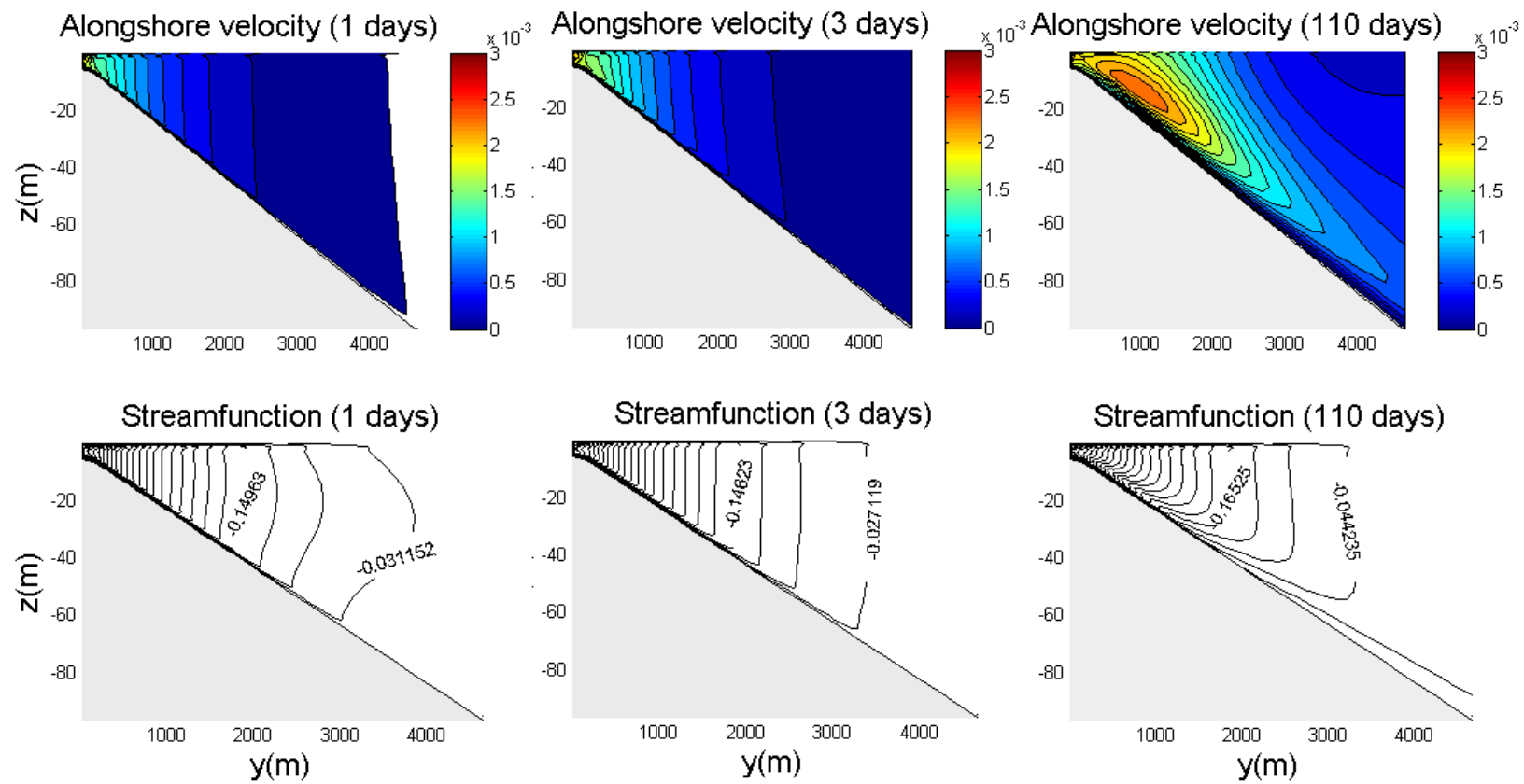

Figure 3.2. Time evolution of the flow in the base case. The fields presented are (top to bottom) temperature, alongshore velocity and cross-shore streamfunction (normalized by $\tau_{0} / \rho f$ ) fields after (A) 1day, (B) 3days and $(\mathrm{C})$ 110days. The contour step for temperature is $0.5^{\circ} \mathrm{C}$. Note the difference in the horizontal axes for the alongshore velocity. 


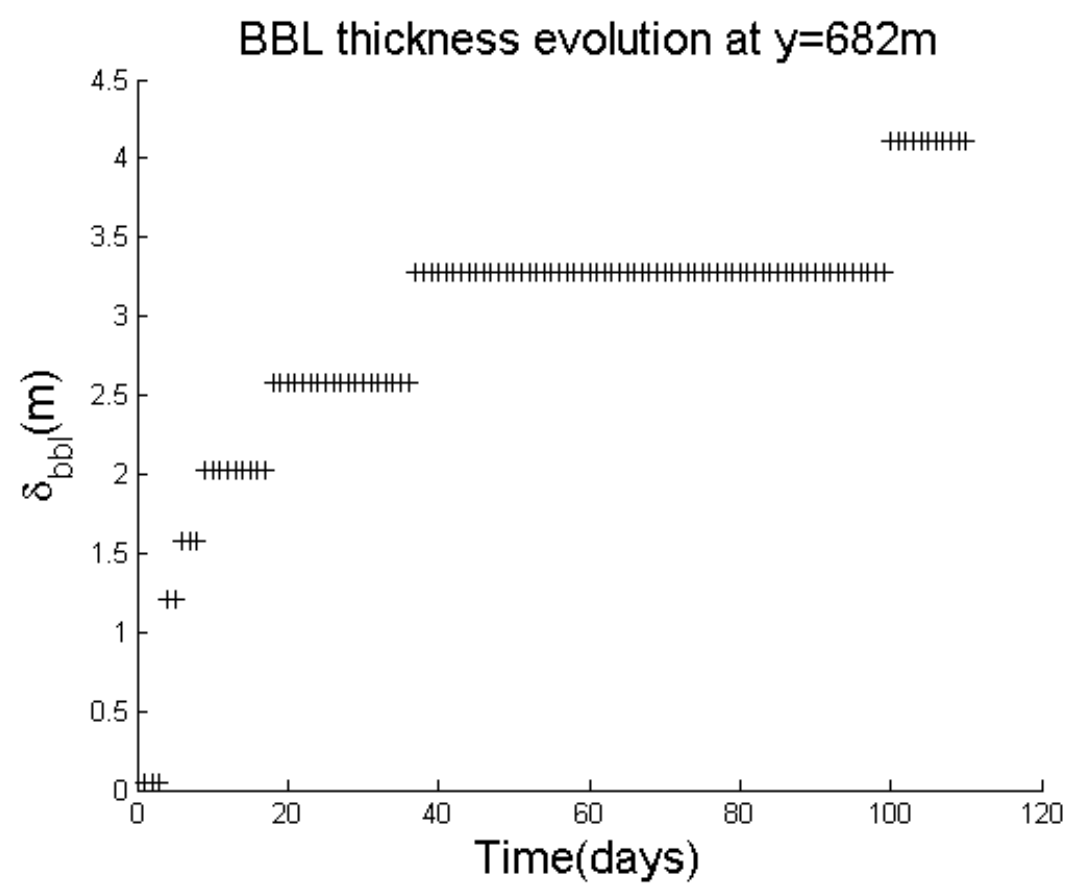

Figure 3.3. The bbl thickness evolution $682 \mathrm{~m}$ offshore. A finite grid size causes step-like appearance of the plot. Jumps in the thickness are equal to the difference between adjacent vertical levels of the grid.
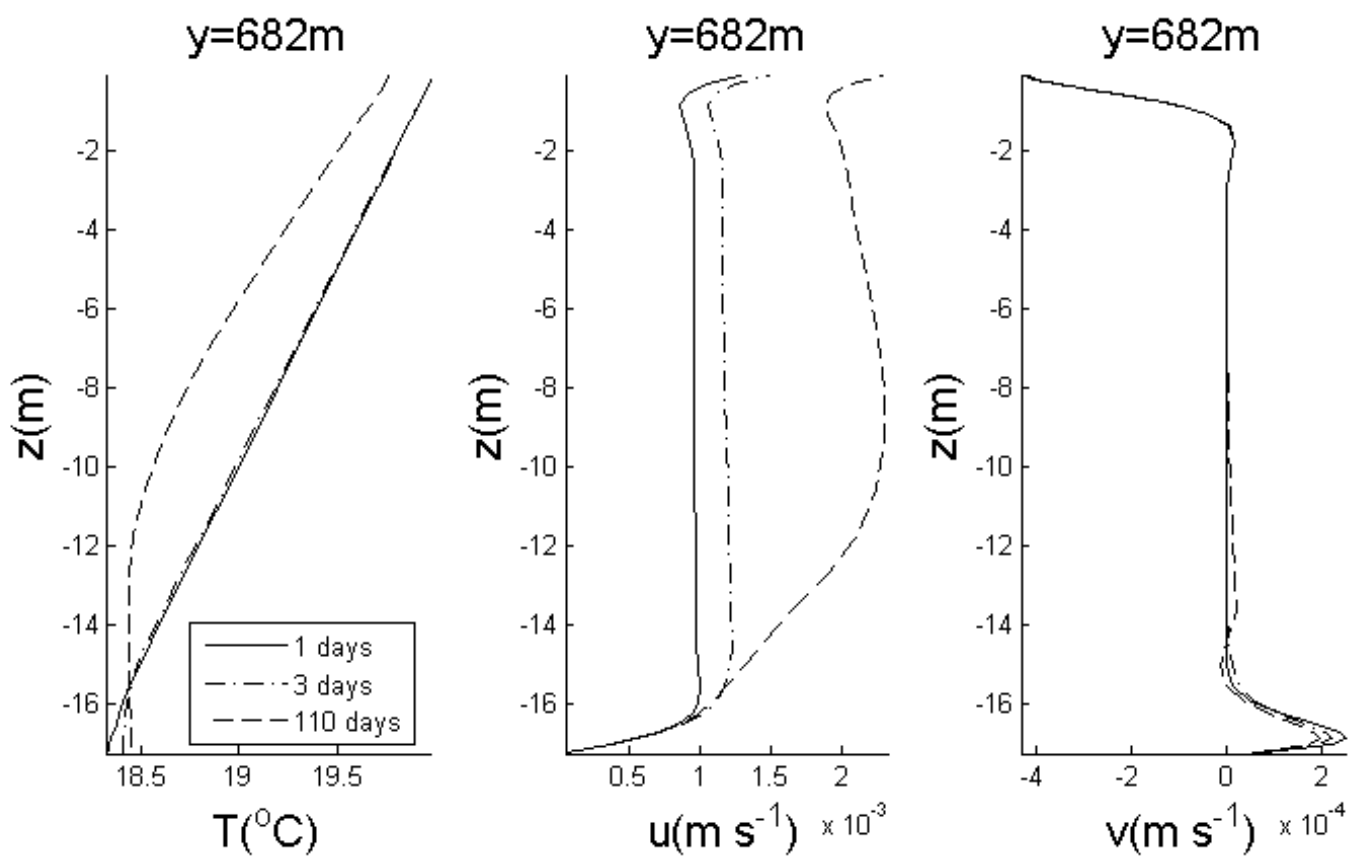

Figure 3.4. (Left to right) The temperature, the alongshore and the cross-shore velocity profiles from bc experiment 682m offshore after 1 day (solid), 3days(dash-dotted) and 110 days(dashed). 
Unfortunately due to computational time constrains the experiment was not ran long enough to reach a complete steady state. In the interior, the time dependence is significant. The main balance here is between the time rate of temperature change and vertical advection of temperature. In the interior, buoyancy is advected by the vertical flow caused by Ekman suction, bringing cold water towards the surface. Because the rate of Ekman suction diminishes offshore, the value of the cold temperature anomaly, caused by the vertical advection, also decreases offshore causing a positive offshore temperature gradient to form. According to the thermal wind balance, this gradient corresponds to a negative shear in the alongshore velocity (Figure 3.4). The presence of a positive vertical shear in the bbl and negative vertical shear in the interior corresponds to the formation of the alongshore velocity maximum at a distance of about a bbl thickness above the bottom.

\subsubsection{Final state}

Although after 110 days the system did not reach a complete steady state, the rate of change of system parameters in the bbl is considered small based on the bbl thickness evolution (Figure 3.3). After 110 days the rate of bbl growth is less than $0.3 \%$ a day. Also a good measure of the steady state is the relative importance of time variation term in the buoyancy balance for the bbl (Figure 3.5). After 110 days this term is less than $10 \%$ of the other terms in the buoyancy equation in the bbl.

\section{Balances}

From the experiment output I can evaluate the relative importance of the terms in the momentum and buoyancy balances (Figure 3.5). The observed flow could be divided into several primary layers: the interior, surface Ekman layer $(\sim 2 \mathrm{~m})$ and the bottom boundary layer. 

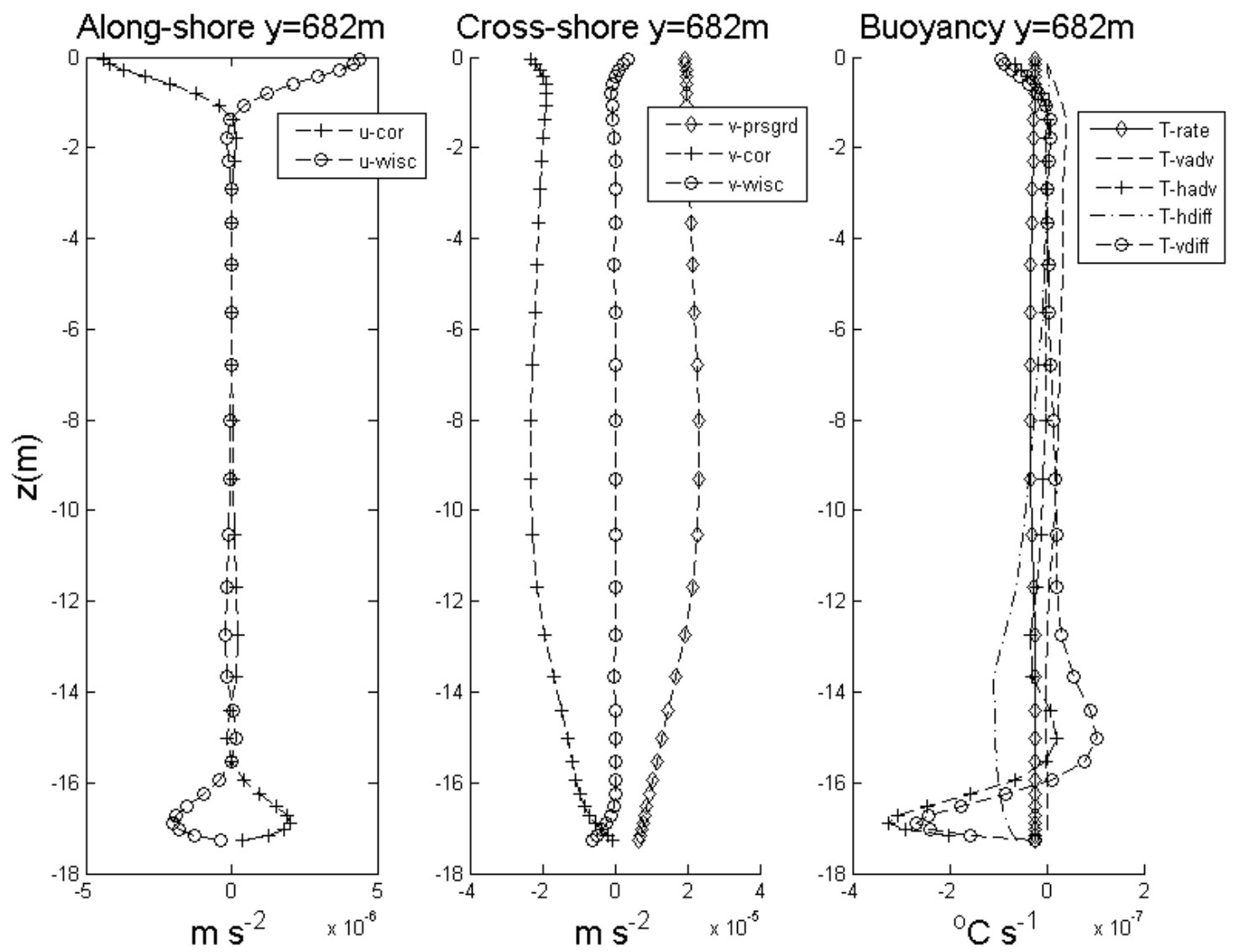

Figure 3.5. The vertical profiles of terms in (left to right) alongshore, cross-shore momentum and buoyancy equations in the base case run at $682 \mathrm{~m}$ offshore after 110 days. The along-shore momentum equation is a balance between the Coriolis term (cross) and the vertical viscosity term (circle). The cross-shore momentum equation is a balance among the pressure (diamond), the Coriolis (cross) and the vertical viscosity terms (circle). The rest of terms in the momentum equations are negligible. In the buoyancy equation, the time rate of change (diamond), the vertical advection (dashed), the horizontal advection (cross), the vertical diffusion (circle) and the horizontal diffusion (dash-dot) terms are important.

\section{Ekman layer}

Near the surface there is a classical Ekman layer, where the momentum balance between vertical viscous, Coriolis and pressure gradient forces holds: 
3.1

$$
-f v=\frac{\partial}{\partial z}\left(A_{V} \frac{\partial u}{\partial z}\right)
$$

3.2

$$
f u=-\frac{1}{\rho_{0}} \frac{\partial p}{\partial y}+\frac{\partial}{\partial z}\left(A_{V} \frac{\partial u}{\partial z}\right)
$$

3.3

$$
v \frac{\partial T}{\partial y}=\frac{\partial}{\partial z}\left(K_{V} \frac{\partial T}{\partial z}\right)
$$

\section{Interior}

The main balances in the interior are as follows:

3.4

$$
-f v=\frac{\partial}{\partial z}\left(A_{V} \frac{\partial u}{\partial z}\right)
$$

3.5

$$
f u=-\frac{1}{\rho_{0}} \frac{\partial p}{\partial y}
$$

3.6

$$
\frac{\partial T}{\partial t}+w \frac{\partial T}{\partial z}=\frac{\partial}{\partial y}\left(K_{H} \frac{\partial T}{\partial y}\right)
$$

Here we are going to take a closer look at the buoyancy balance. The initial uniformly stratified temperature field is altered. As it was mentioned above in the interior isopycnals are distorted from being horizontal, generating horizontal variation in the density field. This gives rise to a lateral diffusion. It was assumed by Pedlosky (2007) that in steady state horizontal diffusion balanced vertical advection. As it was mentioned above the base case experiment did 
not reach a completely steady state and beside the terms mentioned above time rate of temperature change is significant (3.6).

\section{Bottom boundary layer}

The experimental results show that the balances in the bbl are:

3.7

$$
-f v=\frac{\partial}{\partial z}\left(A_{V} \frac{\partial u}{\partial z}\right)
$$

3.8

$$
f u=-\frac{1}{\rho_{0}} \frac{\partial p}{\partial y}+\frac{\partial}{\partial z}\left(A_{V} \frac{\partial u}{\partial z}\right)
$$

3.9

$$
v \frac{\partial T}{\partial y}=\frac{\partial}{\partial y}\left(K_{H} \frac{\partial T}{\partial y}\right)+\frac{\partial}{\partial z}\left(K_{V} \frac{\partial T}{\partial z}\right)
$$

It was observed that in the final state the time dependence in the bbl is small and bbl buoyancy equation transforms into a balance between the vertical and horizontal diffusion and horizontal advection. This result is similar to the assumption made in Garrett et al.(1993) that in a steady state the advective density flux in the bbl is balanced by the diffusive buoyancy flux through the top of the bbl.

In this problem, the structure of the bbl is of great importance. As it was already pointed out above, the alongshore flow heavily depends on the bbl thickness and bbl horizontal temperature gradient.

The final bbl structure for bc experiment is shown below (Figure 3.6). 


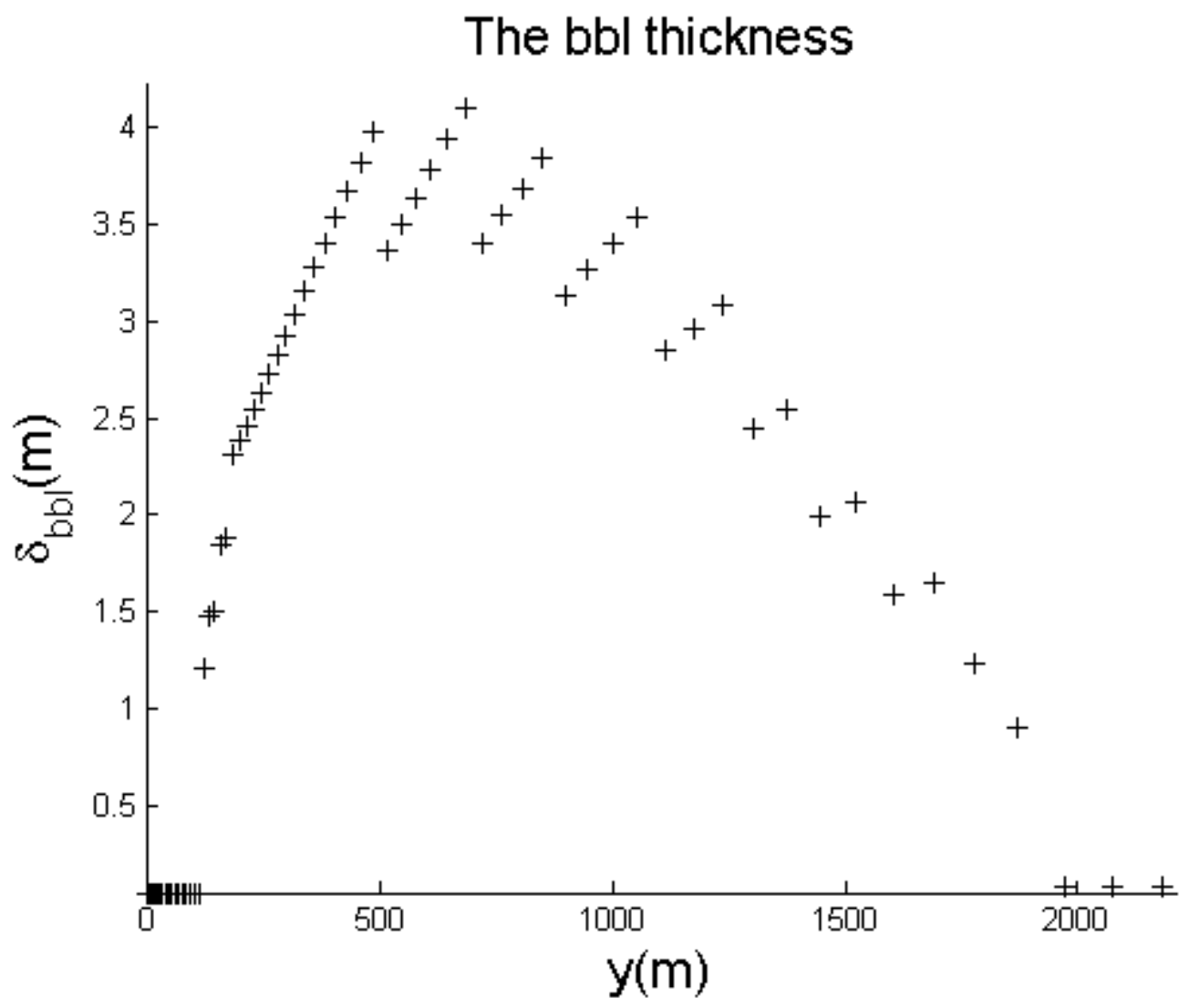

Figure 3.6. The bbl thickness as a function of the offshore position in the bc experiment after 110 days.

The bbl has a horizontal extent of about $2000 \mathrm{~m}$ with a maximum thickness of $3.8 \mathrm{~m}$ near $600 \mathrm{~m}$ offshore. It has a rounded triangular shape with an averaged side slope of $10^{-2}$ inshore and $2 * 10^{-3}$ offshore. The temperature field has a structure similar to the CL model. There they assumed that temperature in the bbl is vertically well mixed, continuous through the top of bbl and thereby has a negative offshore temperature gradient. The magnitude of the horizontal temperature gradient is variable and determined by the interior stratification and the offshore variation in the bbl thickness. Results of the base case numerical experiment demonstrate good agreement with the CL theory. The horizontal temperature gradient has lower values in the inshore part (top of the bbl is more flat) of the bbl and higher values in the offshore part (Figure 
3.7). Throughout the whole lateral extent of the bbl, the horizontal temperature gradient is of the order of $T_{z 0} * \alpha=2 * 10^{-3 \circ} \mathrm{C} \mathrm{m}^{-1}$, where $T_{z 0}$ is the interior stratification and $\alpha$ is the bottom slope.

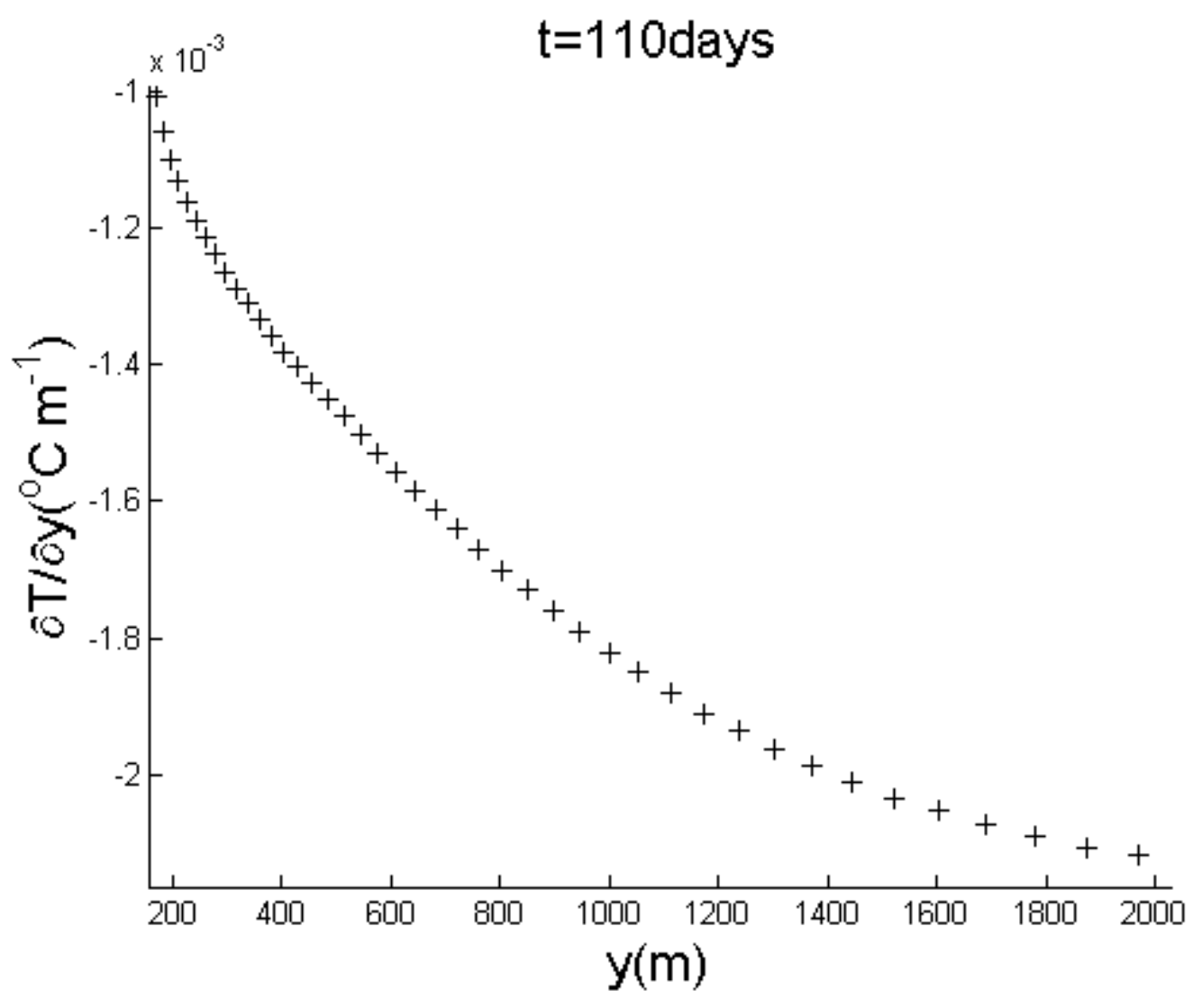

Figure 3.7. Temperature gradient in the bbl as a function of the offshore location after 110 days (from the bc experiment).

\subsubsection{Experiments with one changing parameter}

In this section we are going to investigate the dependence of the bbl behavior on several parameters: the background vertical diffusivity, the initial stratification and the bottom slope. A 
set of numerical experiments was conducted with only one parameter from this list being changed. Due to the computational time constraints the experiments were conducted for time periods of 25 to 37 days. ${ }^{1}$

\subsubsection{Experiments with variable background diffusivity}

Three experiments were conducted in which all the parameters, except for the background vertical diffusivity, were kept the same. I use the following values of background vertical diffusivity: $K_{V_{b a k}}=10^{-6} ; 2 * 10^{-6} ; 10^{-5} \mathrm{~m}^{2} / \mathrm{s}$ (cases bc, $2 K_{v_{b c}}$ and $10 K_{v_{b c}}$ respectively).

The results of the experiment revealed significant dependence of the bbl, and the overall downwelling response of the flow, on the value of the background vertical diffusivity. The cross sections of final states (after 37 days) of the alongshore velocity, cross-shore stream function and temperature fields are presented on Figure 3.8.

\footnotetext{
${ }^{1}$ After these time periods the flow did not reach a completely steady state. However in all the runs in the final state the temperature rate of change in the bbl buoyancy equation is at least 2 times smaller than the other terms.
} 

(A) bc
(B) $2 K_{V_{b c}}$
(C) $10 K_{V_{b c}}$
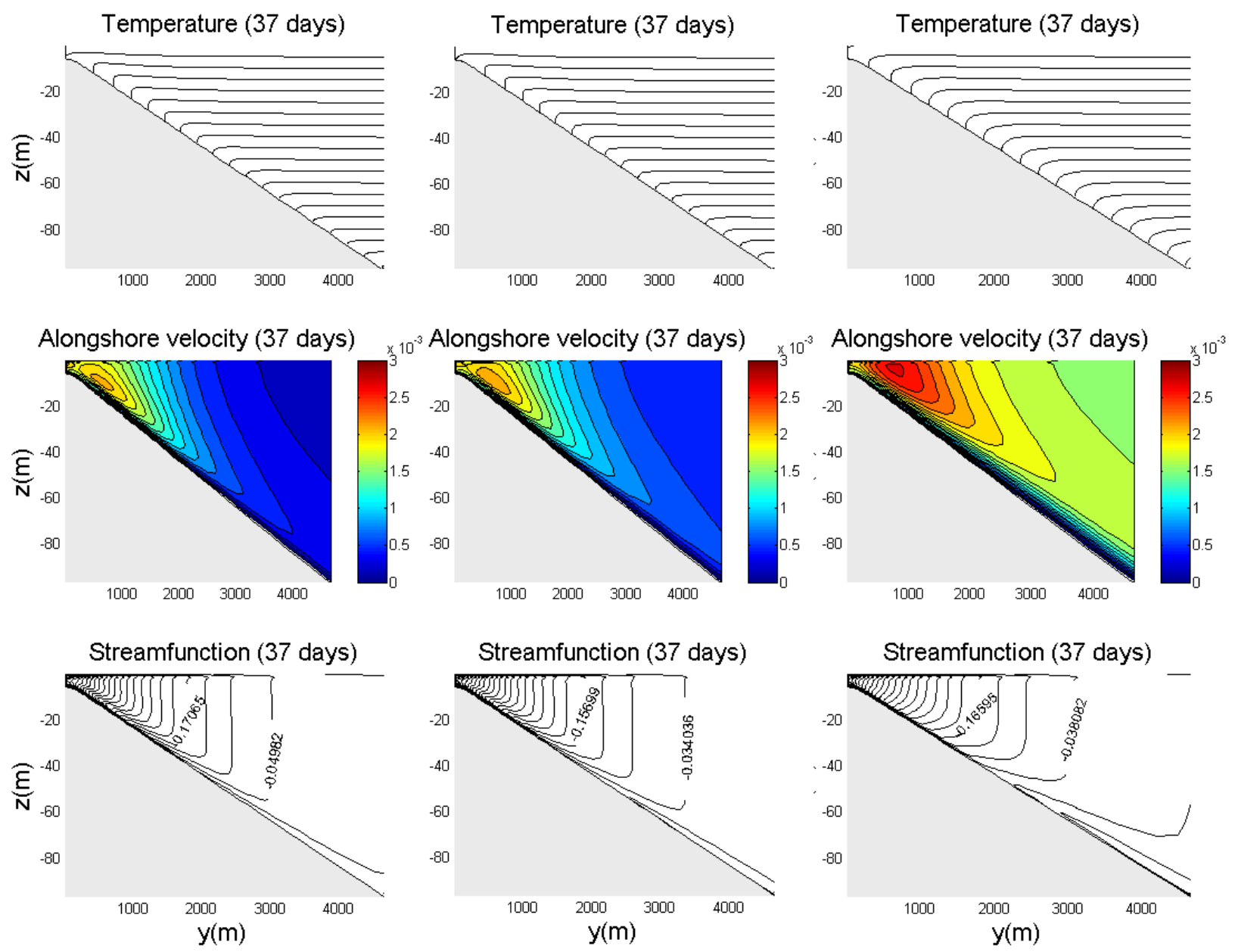

Figure 3.8. The difference in the flow structure in the experiments with variable background vertical diffusivity. Top to bottom: the temperature, the alongshore velocity and the cross-shore streamfunction (normalized by $\tau_{0} / \rho f$ ) fields after 37 days. Columns left to right: (A) base case; (B) $2 K_{V_{b c}}$; (C) $10 K_{V_{b c}}$. The contour step for temperature is $0.5^{\circ} \mathrm{C}$.

First, I am going to take a closer look at the bbl structure. It was observed that the higher values of $K_{V_{b a k}}$ yield the thinner and narrower bbl (Figure 3.9). 


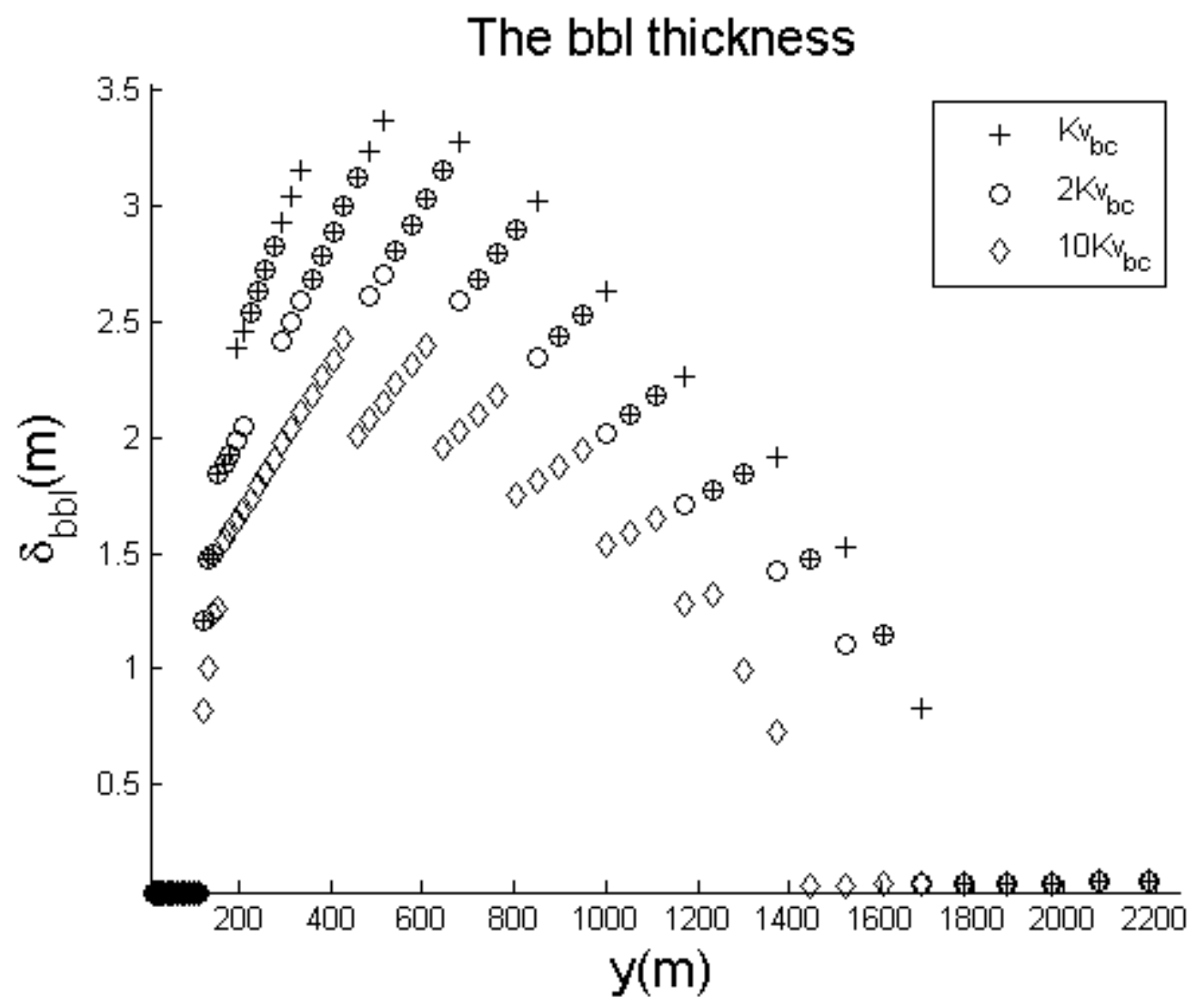

Figure 3.9. The bbl thickness as a function of the offshore location after 37 days from experiments with different values of background vertical diffusivity: bc(cross), $2 K_{V_{b c}}\left(\right.$ circle) and $10 K_{V_{b c}}$ (diamond)

It is in qualitative agreement with result of Pedlosky(2007), where using an analytical model, it was shown that for high values of the interior diffusivity the bbl is thinner and limited in horizontal extent (Figure 3.10). 


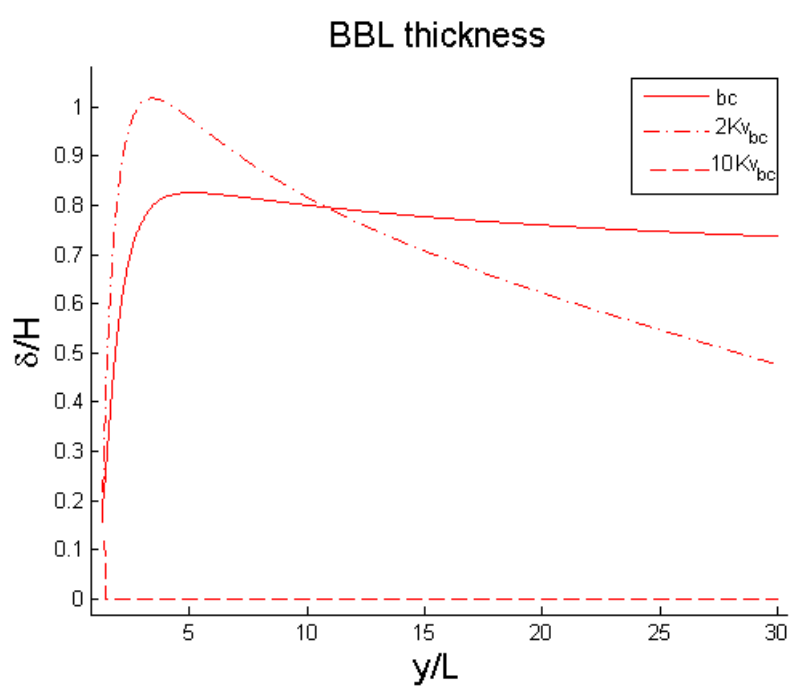

Figure 3.10. Analytical solution (Pedlosky, 2007) for bbl thickness using the same parameters as in the experiments bc (solid), $2 K_{V_{b c}}$ (dash-dot) and $10 K_{V_{b c}}$ (dash). The values are in non-dimensional units. $H$ is a local depth and $L=L_{W}$ is the horizontal wind scale.

It was observed that the higher the value of the background diffusivity, the smoother the transition in temperature between the linearly stratified interior and the vertically well mixed bbl (Figure 3.11, left).

This suggests the presence of another diffusive boundary layer $(\mathrm{dbl})$, thickness of which is proportional to the background diffusivity. In this layer, the buoyancy time rate of change is balanced by horizontal and vertical diffusion (Figure 3.12):

3.10

$$
\frac{\partial T}{\partial t}=K_{V} \frac{\partial^{2} T}{\partial z^{2}}+K_{H} \frac{\partial^{2} T}{\partial y^{2}}
$$



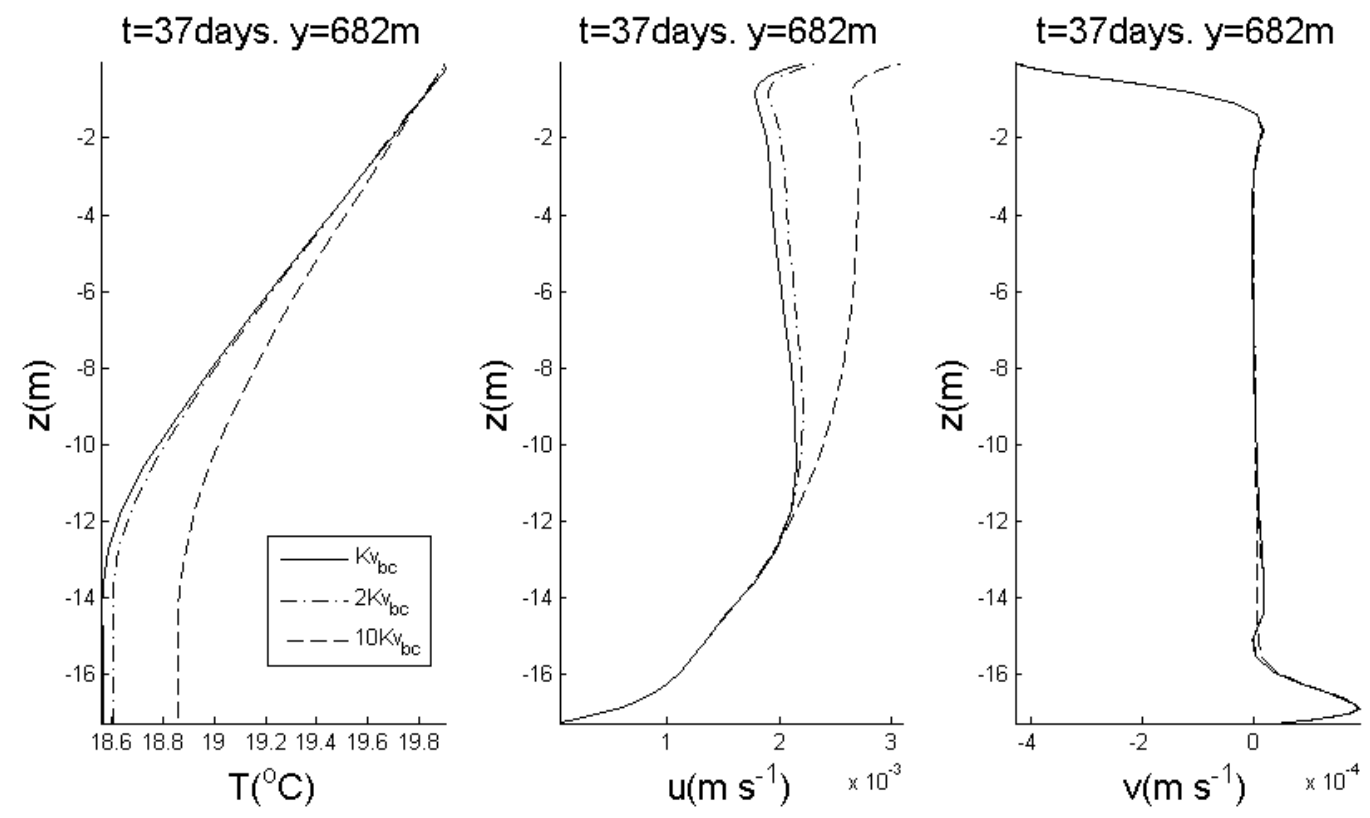

Figure 3.11. Left to right: the temperature, the alongshore velocity and the cross-shore streamfunction vertical profiles $682 \mathrm{~m}$ offshore from the experiments with variable background vertical diffusivity.

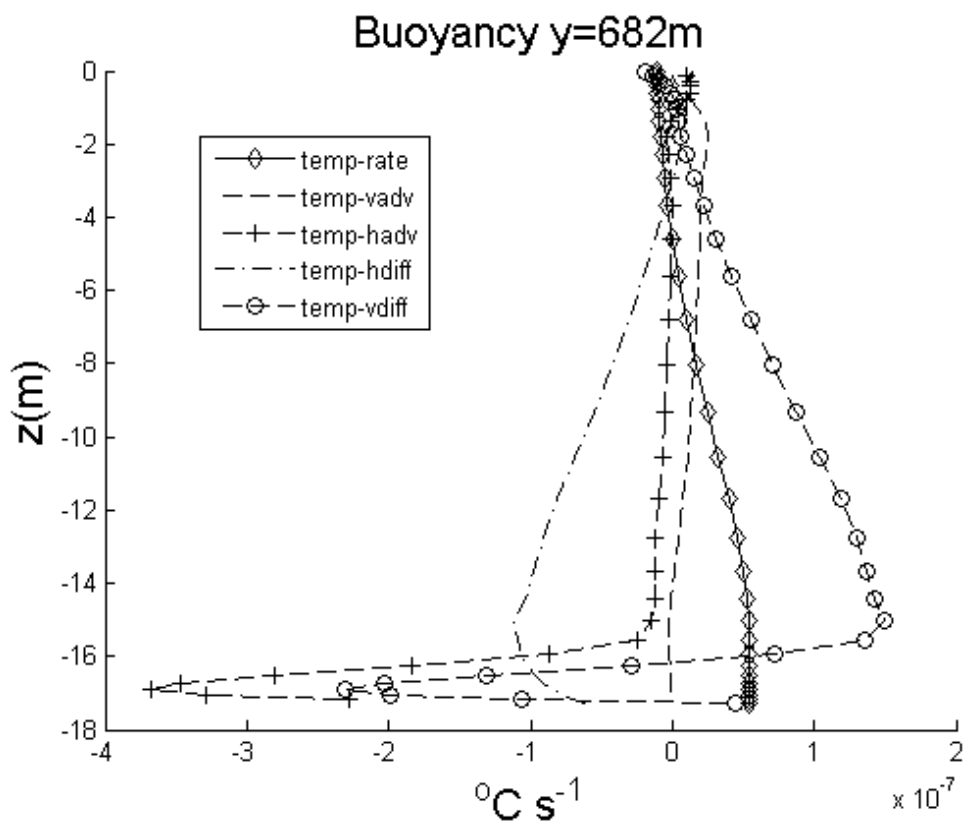

Figure 3.12. The buoyancy equation terms from experiment $10 K_{V_{b c}} 682 \mathrm{~m}$ offshore after 37 days. Terms shown are the time rate of change (diamond), the vertical advection (dashed), the horizontal advection (cross), the vertical diffusion (circle) and the horizontal diffusion (dash-dot) terms. 
Therefore in this case a double bottom boundary layer structure is present. In dbl, the ambient stratification is altered by diffusive processes and, due to the sloping bottom, a negative horizontal cross shore gradient of temperature is formed. This in turn, through the thermal wind balance, supports a positive vertical shear in the along-shore velocity. A higher $K_{V_{b a k}}$ results in a thicker diffusive layer, and a greater area of positive shear results in a higher maximum alongshore velocity.

The along-shore velocity in the first two experiments ( $b c$ and $2 K_{V_{b c}}$ ) has a maximum near the bottom and in the experiment $10 K_{V_{b c}}$ it still has a positive shear above the bbl due to the presence of a dbl ( Figure 3.11).

Stream function fields look similar, with the offshore volume transport less concentrated near the bottom for the higher values of $K_{V_{b c}}$.

\subsubsection{Experiments with variable initial stratification}

I conducted two experiments with an initial stratification of $T_{z 0}=0.1^{\circ} \mathrm{C} \mathrm{m}^{-1}$ and $0.2^{\circ} \mathrm{C} \mathrm{m}^{-1}$ (surface heat flux is adjusted accordingly) (experiments bc and $2 N_{b c}^{2}$ ). The model ran for 25 days. The final state of the flow is shown on (Figure 3.13) 


$$
\text { (A) } b c
$$

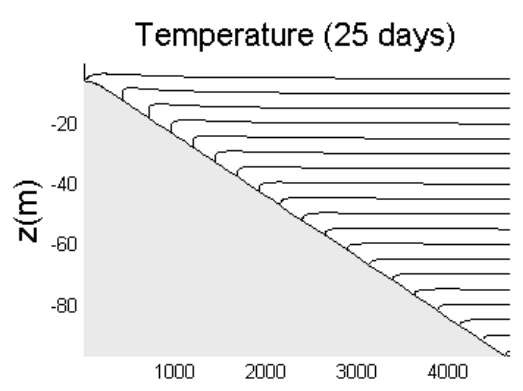

(B) $2 N_{b c}^{2}$

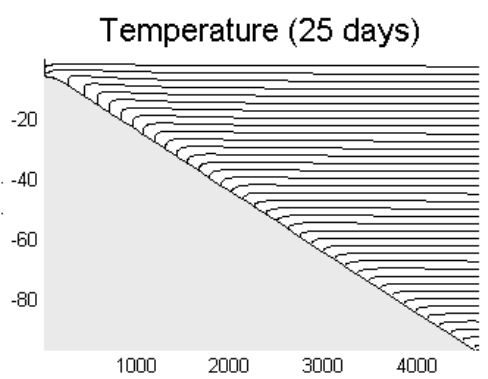

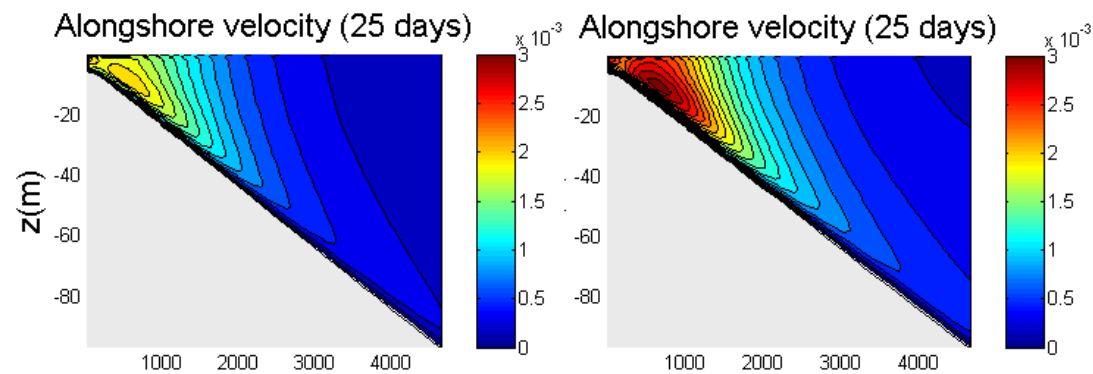
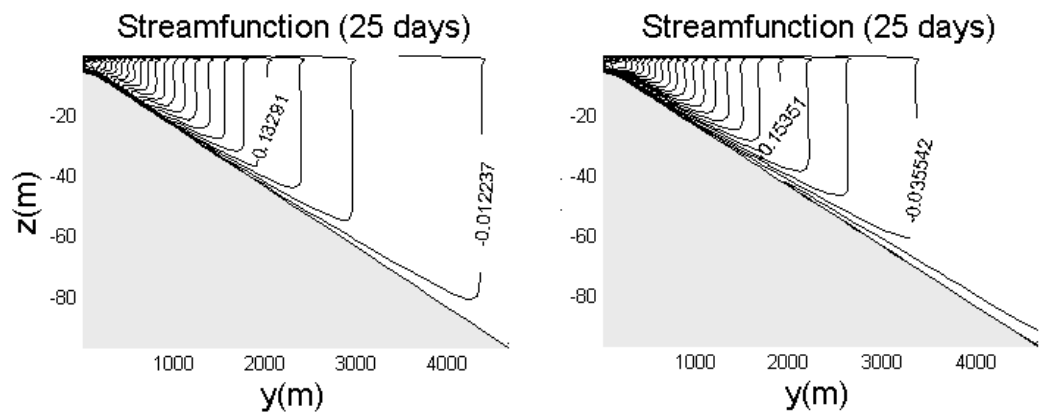

Figure 3.13. The difference in the flow structure in the experiments with variable initial stratification. (Top to bottom) the temperature, thee alongshore velocity and the cross-shore stream function fields after 25 days. Columns: (A) base case; (B) $2 N_{b c}^{2}$. The contour step for temperature is $0.5^{\circ} \mathrm{C}$. The alongshore velocity is much higher in the $2 N_{b c}^{2}$ case.

First I am going to analyze the bbl structure in this case. The bbl in case $2 N_{b c}^{2}$ is thinner and has smaller horizontal extent than in the base case (Figure 3.14). 


\section{The bbl thickness}

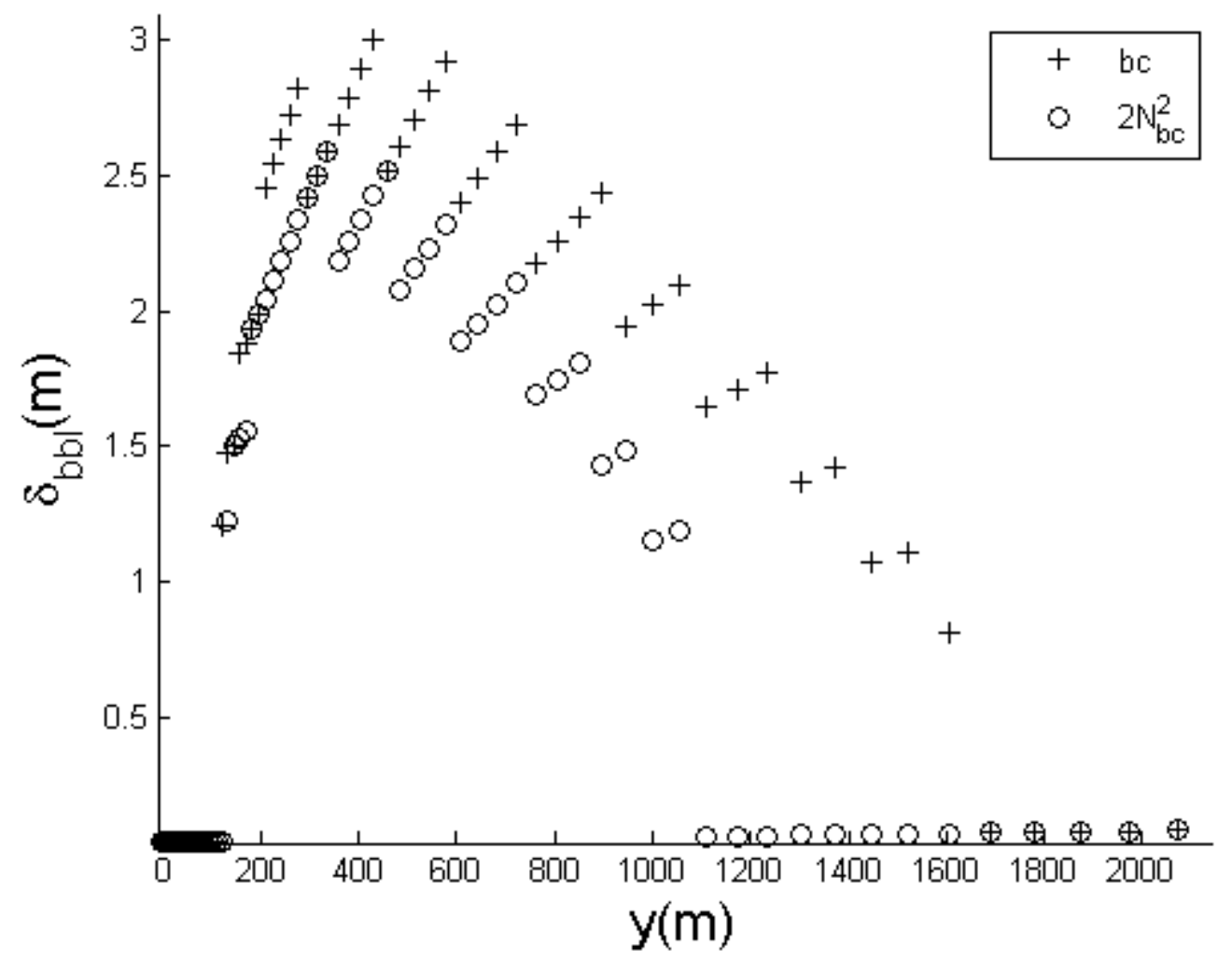

Figure 3.14. The bbl thickness as a function of the offshore location after 25 days from the experiments with different initial stratification: bc (cross) and $2 N_{b c}^{2}$ (circle);

However as the interior stratification is twice stronger in the former than in the latter case, the offshore temperature gradient in the bbl is larger in case $2 N_{b c}^{2}$ (it is of the order of $\alpha T_{z 0}$ ).

It is easy to observe that the alongshore velocity is greater in case $2 N_{b c}^{2}$ (Figure 3.15). This is not surprising. Alongshore velocity is geostrophic. Thereby the alongshore velocity shear could be obtained using the thermal wind relationship. To get the value of the alongshore velocity at the point of maximum, one needs to integrate the obtained shear over the bbl thickness. Though bbl is somewhat thicker in bc experiment the horizontal temperature gradient (and the shear) is twice as big in the $2 N_{b c}^{2}$ case. 
The cross-shore circulation has a similar structure in both case, however the offshore transport in the $2 N_{b c}^{2}$ case is more localized near the bottom (Figure 3.15).
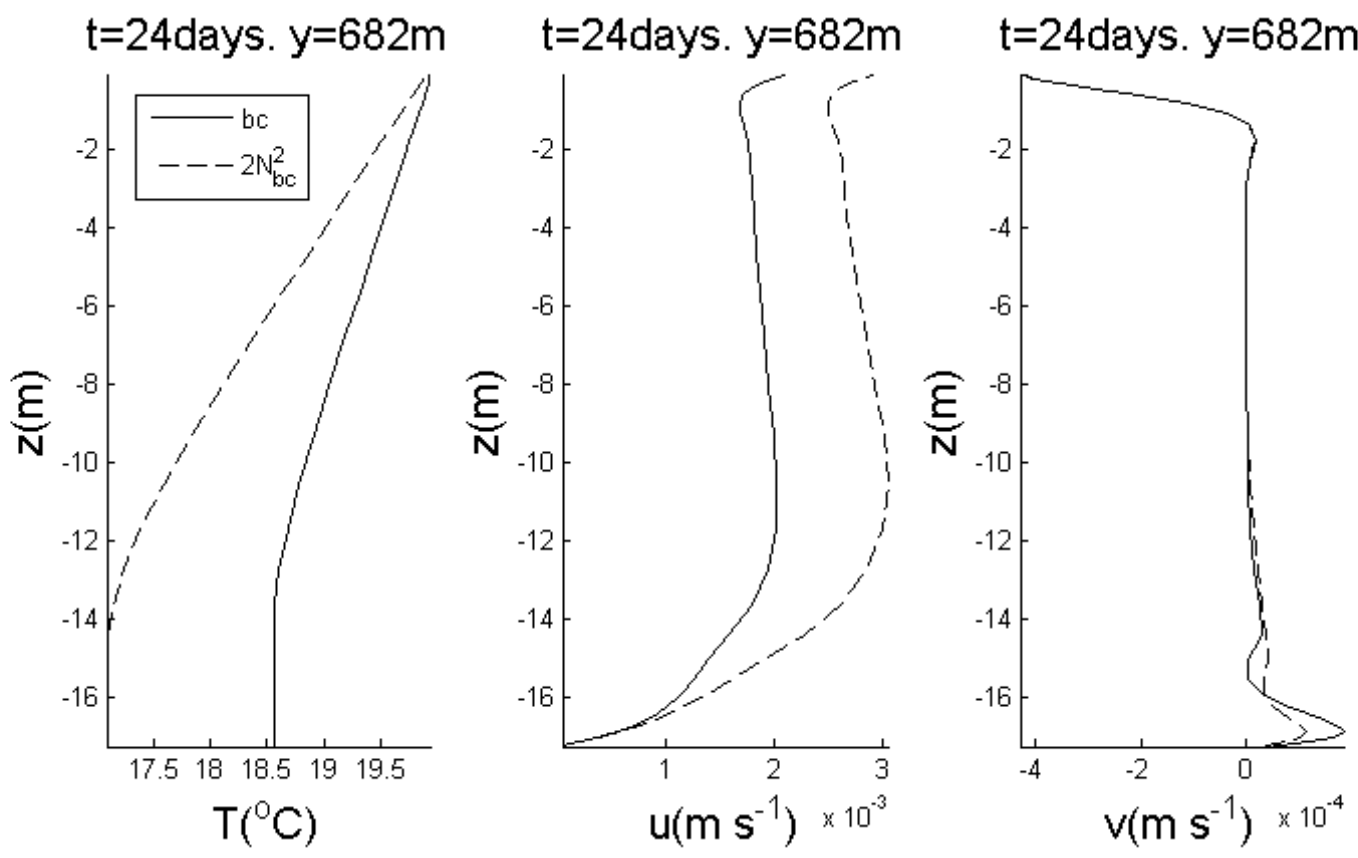

Figure 3.15. (Left to right) The temperature, the alongshore velocity and the cross-shore stream function vertical profiles from the experiments with variable initial stratification: bc (solid) and $2 N_{b c}^{2}$ (dashed) experiments 682m offshore.

\subsubsection{Experiments with variable bottom slope}

In this section we investigated the dependence of the downwelling response on the bottom slope. Results from three experiments with a variable bottom slope of values $2 *$ $10^{-2}, 10^{-2}$ and $2.4 * 10^{-3}$ (experiments: bc, $\alpha_{b c} / 2$ and $\alpha_{b c} / 8.3$ respectively) are presented here. All other parameters were kept the same as in a base case and the model was run for 25 days.

There are significant differences between the results of these three runs (Figure 3.16). 

(A) $b c$
(B) $\alpha_{b c} / 2$
(C) $\alpha_{b c} / 8.3$
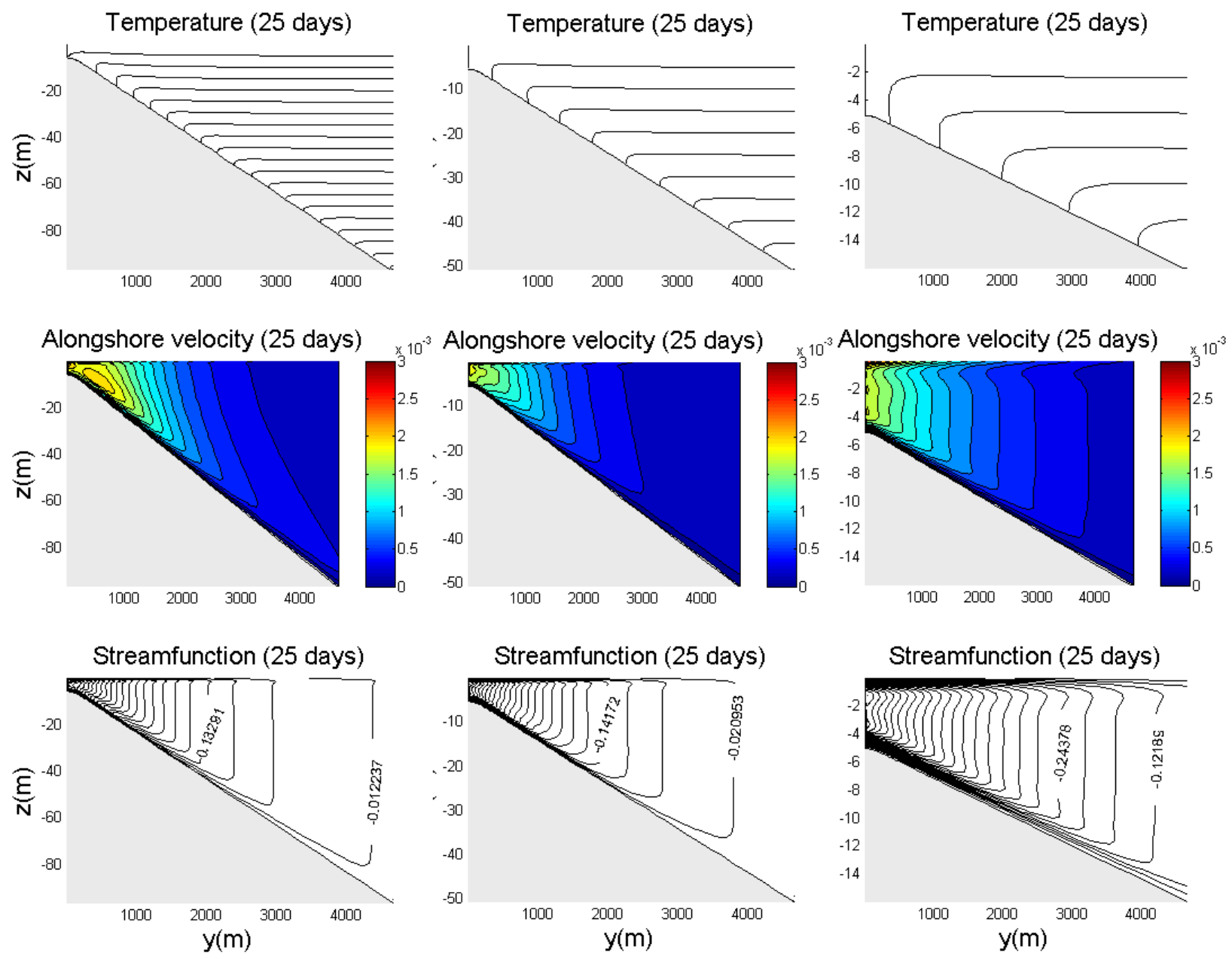

Figure 3.16. The difference in the flow structure in the experiments with variable bottom slope. Top to bottom: temperature, alongshore velocity and cross-shore stream function fields after 25 days. Left to right: (A) base case, (B) $\alpha_{b c} / 2$ and (C) $\alpha_{b c} / 8$. 3. The contour step for temperature is $0.5^{\circ} \mathrm{C}$ for $(\mathrm{A}, \mathrm{B})$ and $0.25^{\circ} \mathrm{C}$ for $(\mathbf{C})$.

I will start my analysis from the temperature field. The interior has a very similar structure in all cases with the isopycnals having similar slopes. It was observed that the steeper the slope, the wider (offshore) is bbl (Figure 3.17). However, the thickness of bbl relative to the local depth is bigger when the slope is small. 


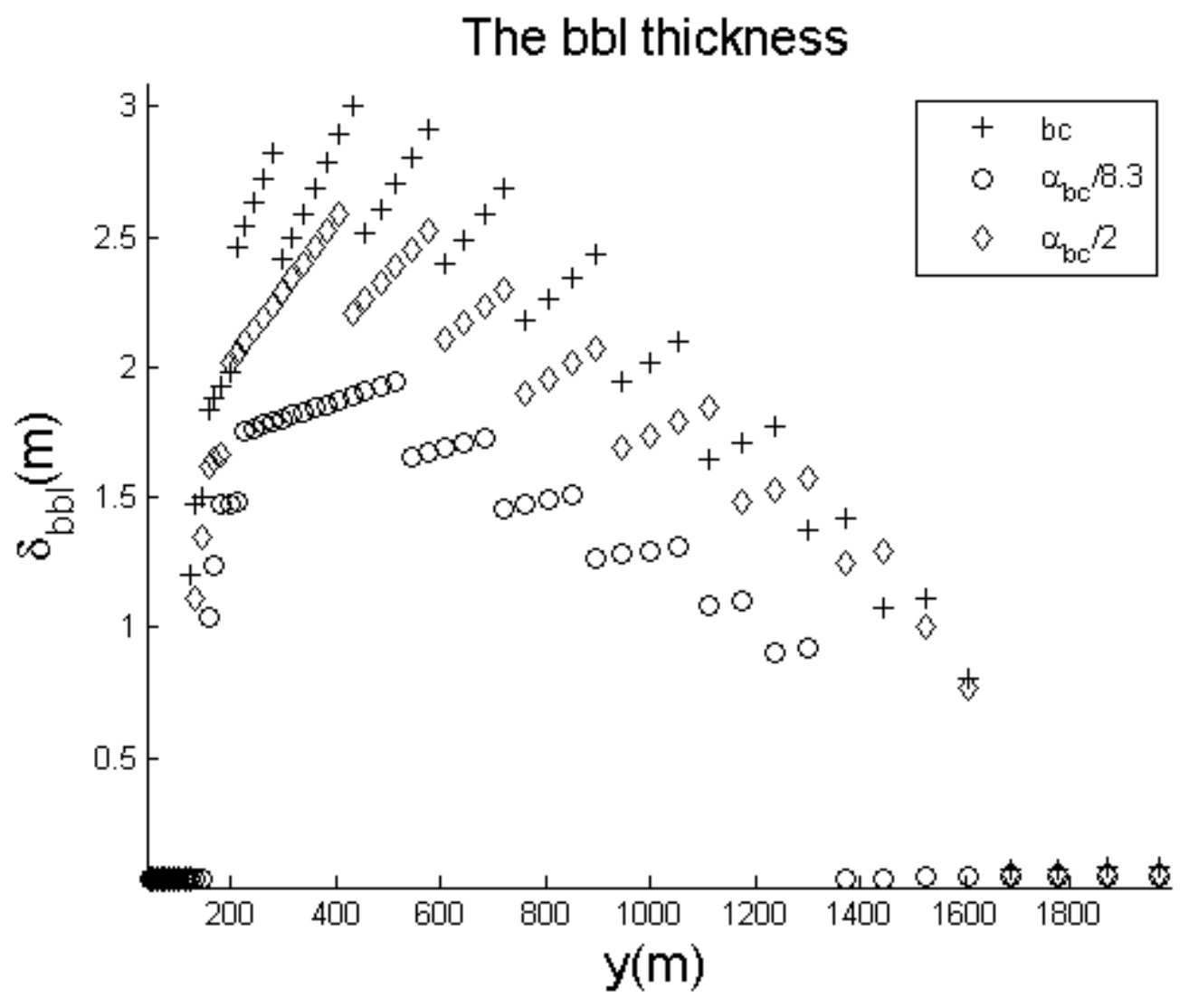

Figure 3.17. The bbl thickness as a function of the offshore location after 25 days from the experiments with variable bottom slope: bc (cross), $\alpha_{b c} / 2$ (diamond) and $\alpha_{b c} / 8.3$ (circle).

The alongshore velocity structure in the different cases has several important differences (Figure 3.18). In the basic case (was discussed above), a maximum velocity is formed at a distance of a bbl thickness above the bottom. The results demonstrate a similar structure in the alongshore velocity in experiment $\alpha_{b c} / 2$, however, it is less intense. There are two reasons for this. First, the bbl offshore temperature gradient is larger in the case of the steeper slope (the gradient is of the order of $T_{z 0} * \alpha$ ). Second, the bbl is thicker in the bc experiment as well, so the shear region is thicker. This leads to a higher alongshore velocity maximum. In the interior, due to the presence of a positive offshore temperature gradient, in both cases alongshore velocity has 
a negative shear. In the case when the slope is much smaller (experiment $\alpha_{b c} / 8.3$ ) the structure is rather different. Due to the shallower depth, the surface Ekman layer and bbl occupy almost the whole depth leaving no room for the interior. In this case velocity field is similar to the homogeneous case.
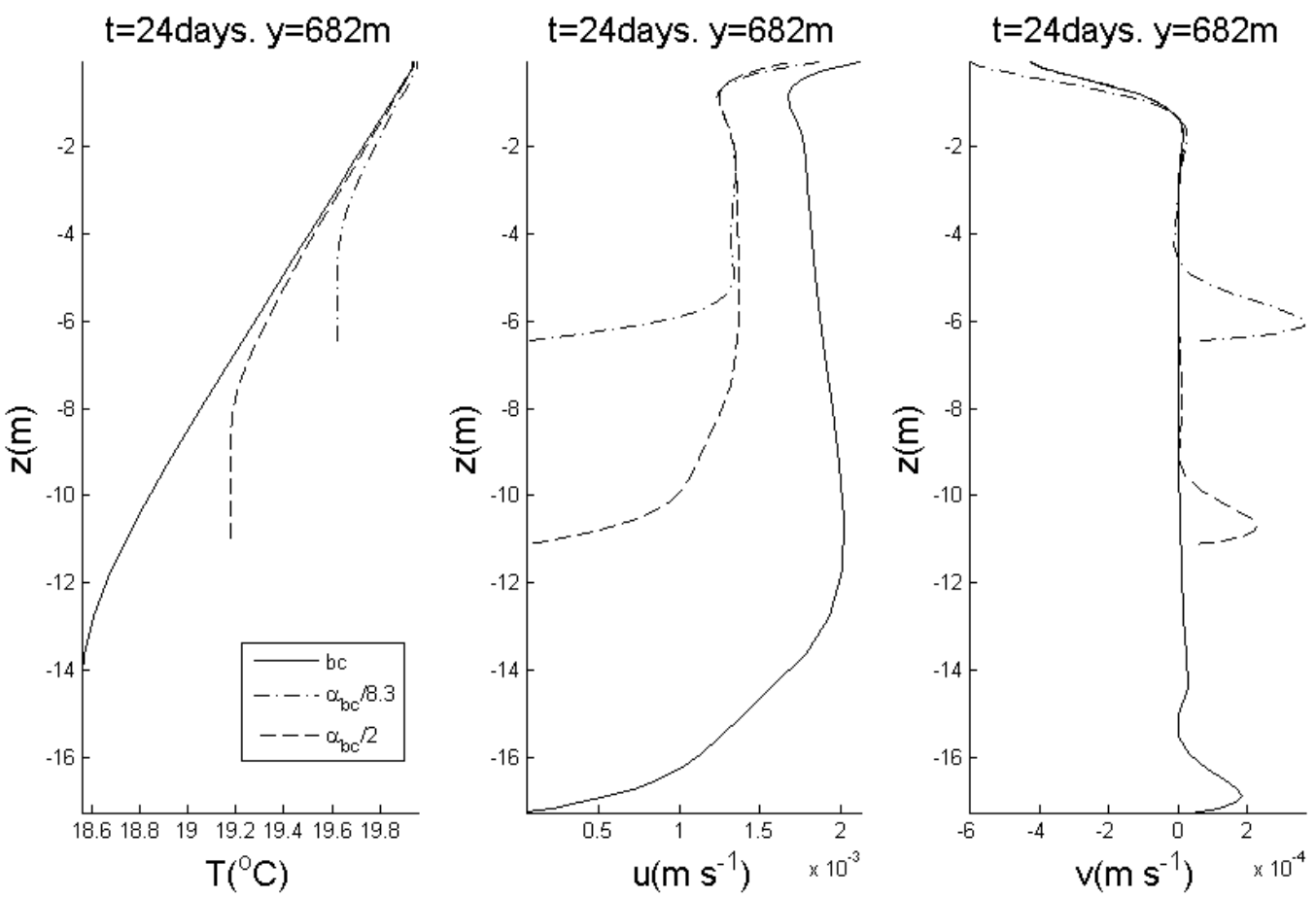

Figure 3.18. (Left to right) The temperature, the alongshore velocity and the cross-shore stream function vertical profiles $682 \mathrm{~m}$ offshore from experiments with variable bottom slope: bc (solid), $\alpha_{b c} / 2$ (dashed) and $\alpha_{b c} / 8.3$ (dash-dot) experiments.

\subsection{Pool case}

This type of a downwelling response takes place when the wind is strong. In this case, the flow has two dynamically different zones: the pool and the mid-shelf, which are separated by the 
front (Figure 3.1,B). A similar type of response was discussed by Allen and Newberger (1996) and Austin and Lentz (2002).

This section proceeds as follows. Subsection 3.3.1 discusses the basic non-dynamical description of the response. Subsection 3.3.2 gives a region by region dynamical description. Subsection 3.3.3 deals with the temporal development of the flow.

\subsubsection{Basic description}

The initial response of the model to the alongshore wind is the acceleration of the surface intensified alongshore flow. This, in turn, accelerates the surface layer onshore due to the Coriolis force, resulting in the formation of a surface Ekman layer within an inertial period. The wind-forced onshore volume transport in the surface layer is initially balanced by a vertically uniform offshore flow but after several inertial periods becomes concentrated near the bottom, suggesting the formation of a bottom boundary layer. In a time period of approximately the advective time scale ( 2days), a weakly stratified region is formed with a strong horizontal temperature gradient at the offshore edge of the pool. This region grows with time, owing to the convective adjustment of the warm pool water pushed by transport in the bottom Ekman layer beneath the front. As the front moves offshore, its speed of propagation as well as intensity is weakening.

In the pool region, the temperature is vertically well mixed and has a weak positive horizontal gradient (Figure 3.19, top). In the frontal region a strong horizontal temperature gradient is present. Offshore of the front, the temperature field structure resembles the bbl case in that it has a positive horizontal gradient in the unstratified bbl and negative horizontal gradient in stably stratified fluid above the bbl. 
The alongshore velocity decays offshore on the horizontal scale corresponding to the diminishing wind scale. The alongshore velocity field has a different vertical structure in different regions (Figure 3.19, middle). In the pool region, the alongshore velocity has an Ekman layer structure both near the bottom and the surface. It also has a slightly positive vertical shear in the interior, caused by the small offshore temperature gradient. In the vicinity of the front, the interior velocity has a much higher shear. In the mid-shelf region the velocity field has a structure very similar to the bbl case in that it has an Ekman layer variation near the surface, a slightly negative vertical shear in the interior and a positive shear in the bbl.

The cross-shore streamfunction field structure remains the same throughout the whole lateral extension of the shelf (Figure 3.19, bottom). In the interior, the transport is almost vertical, with the scale of the Ekman pumping. Near the surface and bottom, it has Ekman layer like structure, which is slightly altered by the presence of the temperature gradients. 

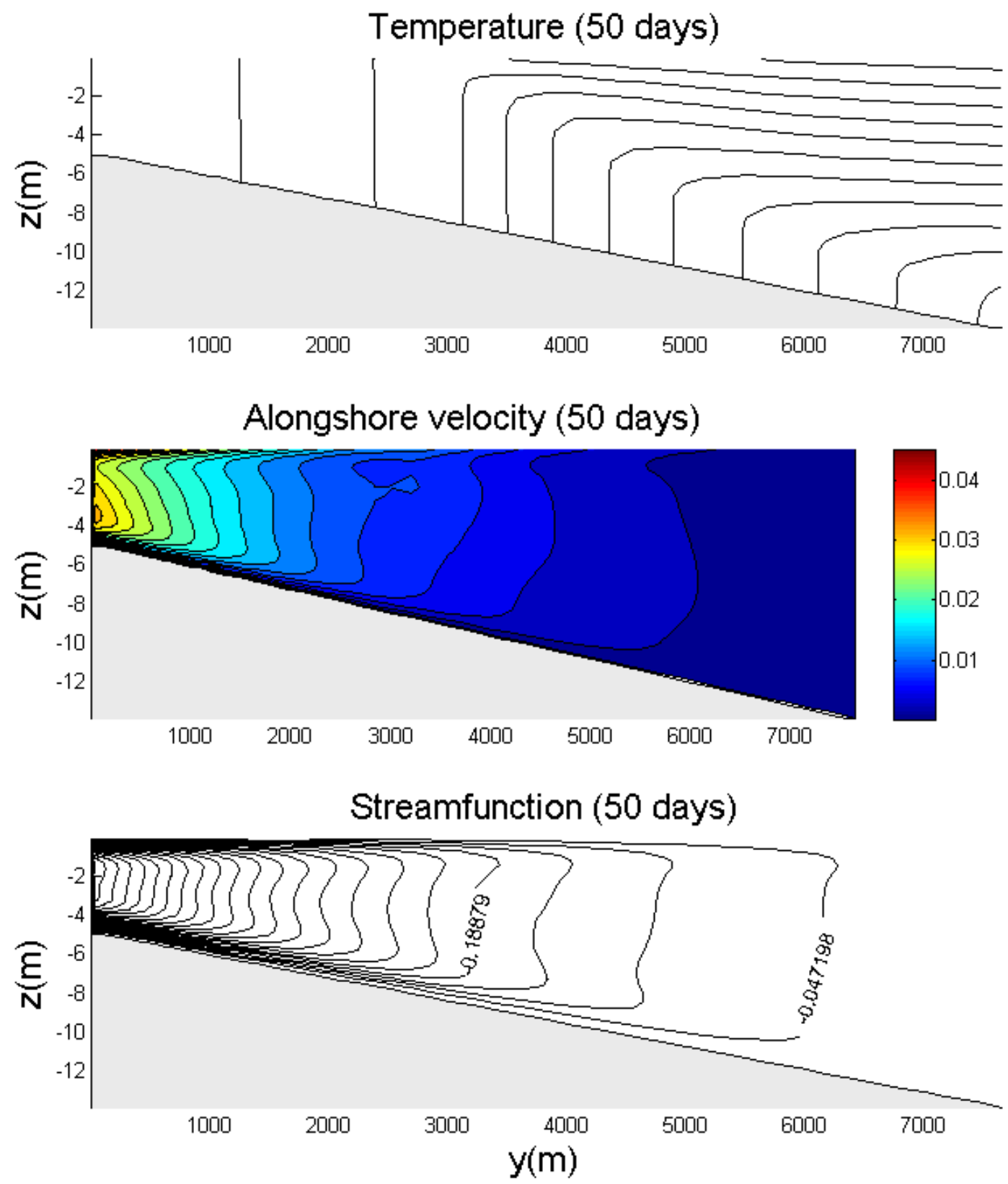

Figure 3.19. The Temperature, the alongshore velocity and the cross-channel streamfunction fields from the pool experiment after 50 days. The contour step for temperature is $1^{\circ} \mathrm{C}$. Note the difference in the horizontal axis for the alongshore velocity. 


\subsubsection{Regional dynamics}

As it was mentioned above we consider three dynamically different regions: the pool, the front and the mid-shelf. The momentum balances in all three regions are the same and will be discussed only for the pool region. But the buoyancy balances are different and will be demonstrated for each region separately.

\subsubsection{The pool region}

The pool region is an unstratified part of the flow between the coastal wall and the front. The vertically uniform structure is considered to be attributable to the advection of light fluid beneath heavy water in the bottom Ekman layer, which causes static instability and leads to enhanced mixing and vertical homogenization in this region.

The dominant terms of the momentum and buoyancy balances in this region obtained from the numerical output (Figure 3.20), suggest relatively simple dynamics. 


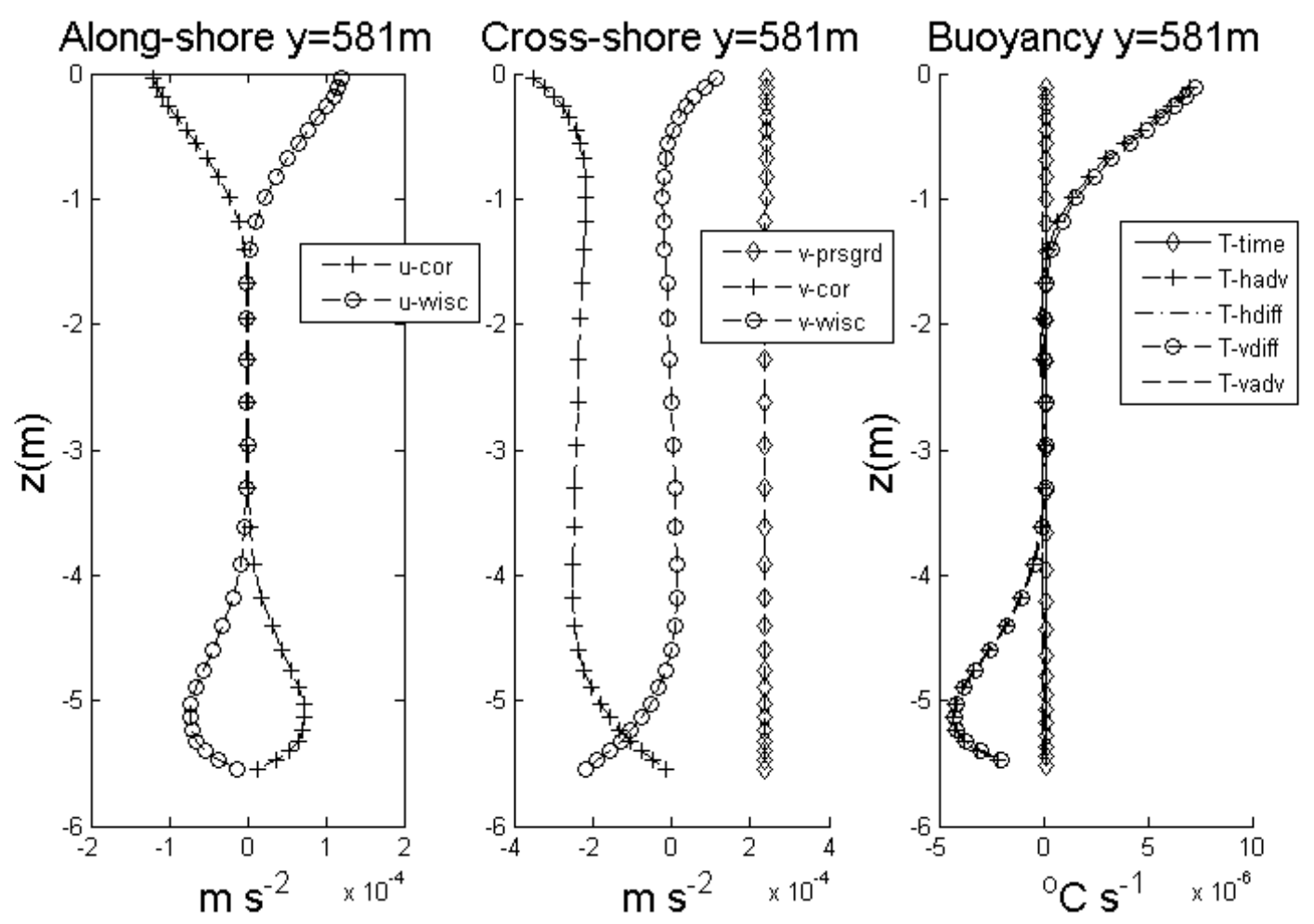

Figure 3.20. Pool region. Terms in the momentum and buoyancy equations from the pool experiment 581m offshore after 50 days. The along-shore momentum equation balance is between the vertical viscosity (circles) and the Coriolis (cross) terms. The cross-shore momentum equation is a balance among the pressure gradient (diamonds), the vertical viscosity (circle) and the Coriolis (cross) terms. In the buoyancy equation the main balance is between the vertical diffusion (circle) and the horizontal advection (cross) terms.

The momentum balance is between the stress divergence term and the Coriolis term, plus a surface pressure gradient. The buoyancy balance is between horizontal advection and vertical diffusion. To a good approximation, the momentum and buoyancy equations in the pool region are:

3.11

$$
-f v=\frac{\partial}{\partial z}\left(A_{v} \frac{\partial u}{\partial z}\right)
$$




$$
f u=-\frac{1}{\rho} \frac{\partial p}{\partial y}+\frac{\partial}{\partial z}\left(A_{v} \frac{\partial v}{\partial z}\right)
$$

3.13

$$
v \frac{\partial T}{\partial y}=\frac{\partial}{\partial z}\left(K_{v} \frac{\partial T}{\partial z}\right)
$$

The flow has Ekman layers both near the surface and the bottom and a geostrophic interior. In the pool experiment, the surface and bottom Ekman layers do not interact, which is different from the case considered by Austin and Lentz (2002), where in the inner shelf region (analog to the pool region here) the surface and the bottom Ekman layers did interact, because the depth of the region in their study is three Ekman layer scales or less (in the current study it is nine Ekman layer scales or more ).

\subsubsection{Frontal region}

The front separates the inner shelf from the mid shelf. This region is characterized by the strongest horizontal density gradient anywhere on the shelf during downwelling and a significant shear in the alongshore flows.

The profiles of the momentum and density balance terms (Figure 3.21) show that the balances are similar to the pool region.

The balances in the along- and the cross-shore momentum equations are the same as in the pool region. The buoyancy balance differs from the one in the pool region, in the frontal region the time rate of change in temperature becomes important. This is attributed to the fact that the front moves offshore. To a good approximation the buoyancy equation in this region is: 


$$
\frac{\partial T}{\partial t}+v \frac{\partial T}{\partial y}=\frac{\partial}{\partial z}\left(K_{v} \frac{\partial T}{\partial z}\right)
$$
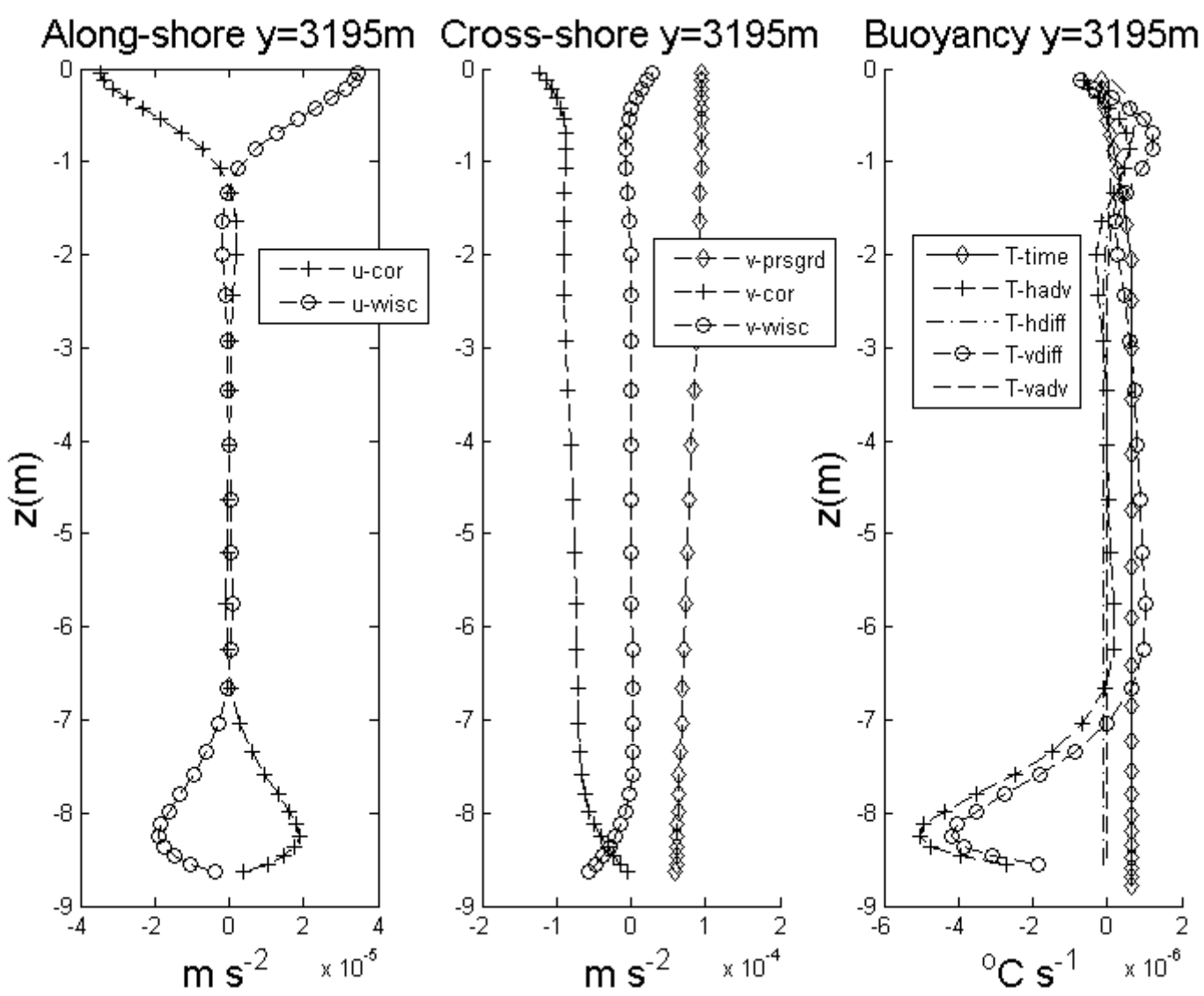

Figure 3.21. The momentum and buoyancy equation balances at the frontal location after 50 days, 3195m offshore. The along-shore momentum equation balance is between the vertical viscosity (circles) and the Coriolis (cross) terms. The cross-shore momentum equation is a balance among the pressure gradient (diamonds), the vertical viscosity (circle) and the Coriolis (cross) terms. In the buoyancy equation the main balance is among the vertical diffusion (circle), the horizontal advection (cross) and the time rate of change (diamond) terms. 


\subsubsection{The mid-shelf region}

This region is right offshore the front. It can also be subdivided into a region where the bbl is present and a region farther offshore where there is no bbl. The dynamics of the former part were discussed in details in the previous section and here I will discuss only the latter part.

The dynamics of this region is driven by the wind and heat flux on the top and the adjusting thermal boundary layer at the bottom. As in the previously discussed regions the momentum balances are primarily geostrophic. Ekman balance holds near the bottom and the surface of the fluid.

The buoyancy balances are slightly different. This region could be vertically separated into two major parts: interior and diffusive bottom boundary layer.

The profiles of terms in the buoyancy equation (Figure 3.22) suggest that the leading balance in the bottom boundary layer is between the time rate of temperature change and vertical diffusion:

3.15

$$
\frac{\partial T}{\partial t}=\frac{\partial}{\partial z}\left(K_{v} \frac{\partial T}{\partial z}\right)
$$

In the interior the balance is between vertical advection and the time rate of temperature change:

3.16

$$
\frac{\partial T}{\partial t}=-w \frac{\partial T}{\partial z}
$$



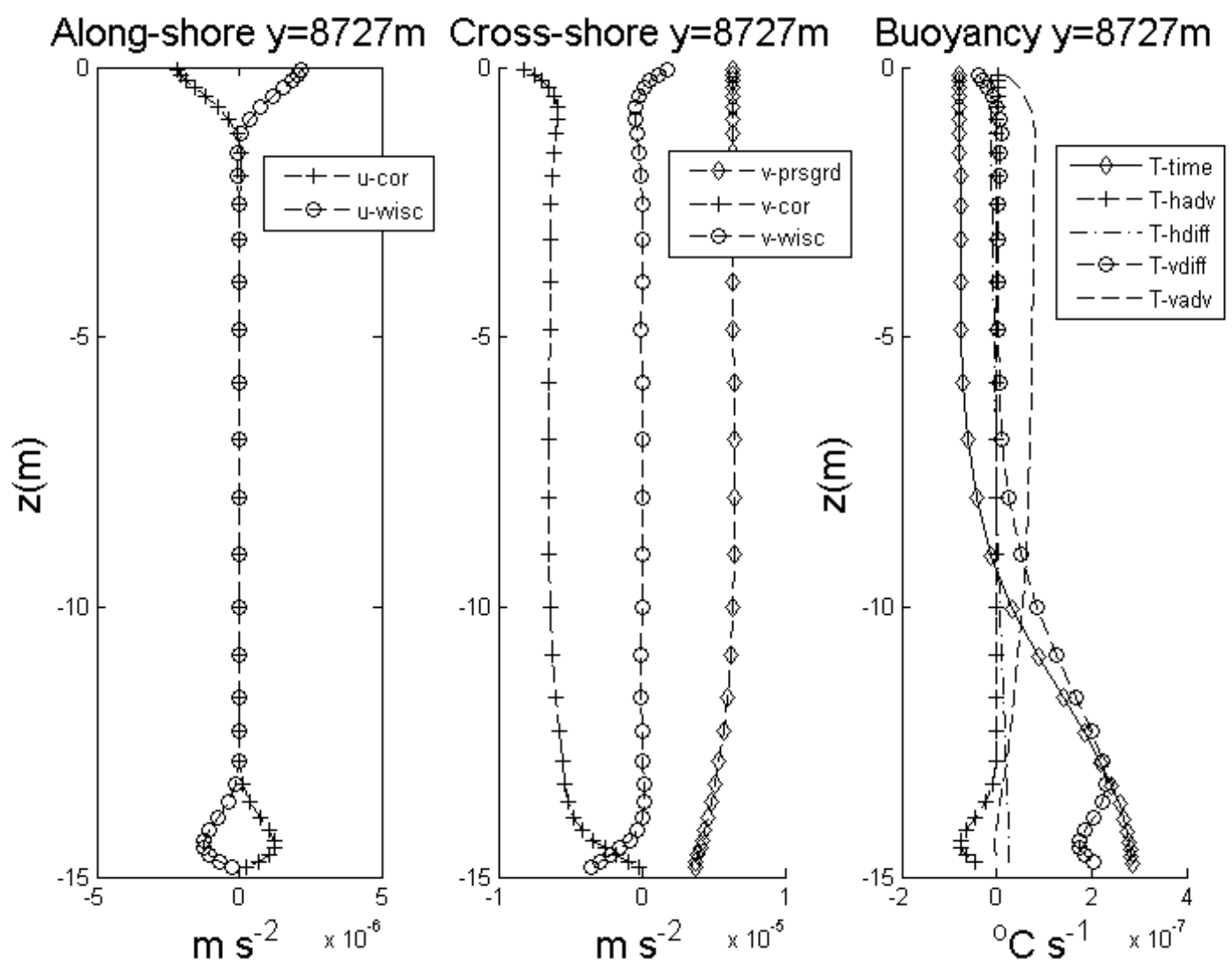

Figure 3.22. The Mid-shelf region. Vertical profiles of the terms in the momentum and the buoyancy equations from the pool case $8727 \mathrm{~m}$ offshore after 50days. The along-shore momentum equation balance is between the vertical viscosity (circles) and the Coriolis (cross) terms. The cross-shore momentum equation is a balance among the pressure gradient (diamonds), the vertical viscosity (circle) and the Coriolis (cross) terms. In the buoyancy equation the main balance is among the vertical diffusion (circle), the vertical advection (dash) and the time rate of change (diamond) terms.

\subsubsection{Temporal development}

In the pool type of response to a downwelling wind the flow is time dependent. In this case the pool region expands (the front moves offshore) with time. The results also demonstrate that as the front moves offshore the horizontal temperature gradient in the front decreases. This time 
dependence of the temperature field corresponds to the temporal evolution of the flow structure. In this section I use an idealized model to better understand the mechanism of the frontal propagation.

\subsubsection{Position of the front}

From the ROMS output it is possible to see that the front propagates offshore with time. In other words, the well mixed inner shelf region expands. This process is determined by the advection of the water in the bottom Ekman layers. In the bottom layer, slightly warmer (lighter) fluid is advected beneath the front which leads to static instability and convective adjustment pushing the edge of the inner shelf further offshore.

To get an estimate of frontal propagation speed, I constructed a simple model based on the mass conservation law, similar to Austin and Lentz (2002). Considering an alongshore section through the front, I assume that the transport in the surface Ekman layer is balanced by barotropic offshore propagation of the front (Figure 3.23). I assume here that the front moves offshore with the same speed throughout the whole water column below the surface Ekman layer. I use the assumption that the convective adjustment time is much smaller than the advective time scale. In other words, the front remains vertical as it moves offshore. 


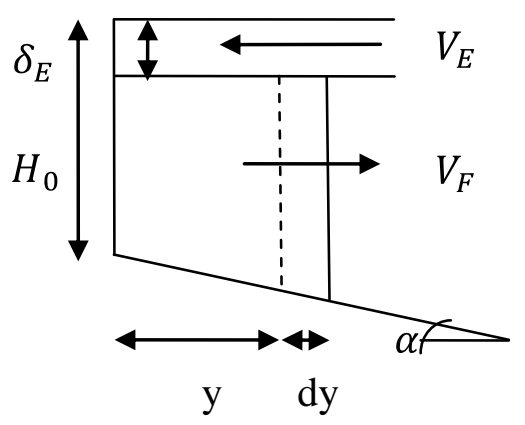

Figure 3.23. The front propagation model where $\delta_{E}$ is an Ekman layer thickness, $V_{E}$ is an Ekman transport, $\alpha$ is the bottom slope, $V_{F}$ is the frontal propagation speed, $H_{0}$ is the depth of the coastal wall, $y$ is the offshore location of the front, $d y$ is an infinitesimal frontal displacement

Using geometry, the area (volume) of the pool region can be expressed as follows:

3.17

$$
A=H_{0} y+\frac{y^{2}}{2} \tan \alpha
$$

The process of pool expansion can be expressed as infinitesimal volume increase:

3.18

$$
V_{E} * d t=d A=\left(H_{0}+y * \tan \alpha\right) * d y
$$

After using the expression for Ekman transport in terms of the wind stress, density and rotation rate and separating the variables the equation is transformed to (also subtracting the area occupied by the Ekman layer):

3.19

$$
\frac{\tau_{0}}{\rho f} * d t=d y *\left(H_{0}-\delta_{E}+y * \alpha\right) * \exp \left(\frac{y}{L}\right)
$$


Integrating the right hand side in time and the left hand side in the offshore coordinate I obtain the moment of time when the front reaches a certain position on the shelf:

3.20

$$
t=\frac{\rho f}{\tau_{0}}\left\{C_{0}+\left[\left(H_{0}-\delta_{E}\right) * L+\alpha *\left(y L-L^{2}\right)\right] * \exp \left(\frac{y}{L}\right)\right\}
$$

Where $C_{0}=-\left[\left(H_{0}-\delta_{E}\right) * L-\alpha * L^{2}\right]$

A comparison between the prediction of this simplified model and the pool experiment data, demonstrates strong agreement (Figure 3.24).

\section{Front propagation}

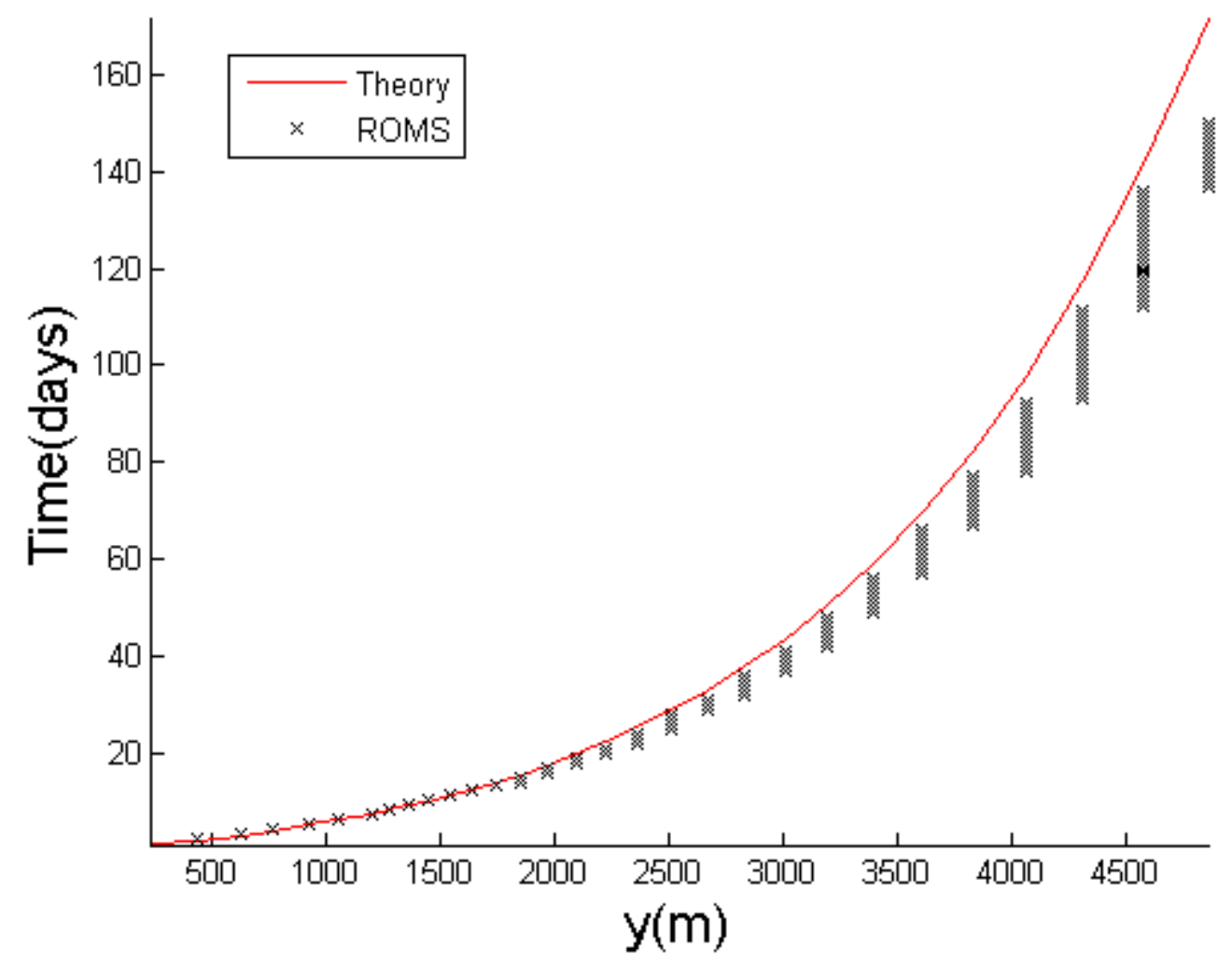

Figure 3.24. Bottom temperature as a function of an offshore position and time. The result from the pool experiment (black) and theoretical model (red) are shown. 
Thereby the time it takes the front to reach a certain location offshore is proportional to the Coriolis parameter and the bottom slope, is inversely proportional to the wind stress amplitude and has a rather complicated dependence on the horizontal e-folding wind scale. The latter suggests that a larger horizontal scale results in a smaller time. So a higher wind stress amplitude, a weaker (slower) offshore variation of the wind (a larger horizontal wind scale), a smaller slope, a smaller Coriolis parameter result in a faster propagation.

\subsubsection{Front intensity}

Data obtained from the numerical experiments demonstrated that the front looses intensity (the frontal temperature gradient decreases) as it moves offshore (Figure 3.25). This was not reported in the previous studies for the constant wind stress.

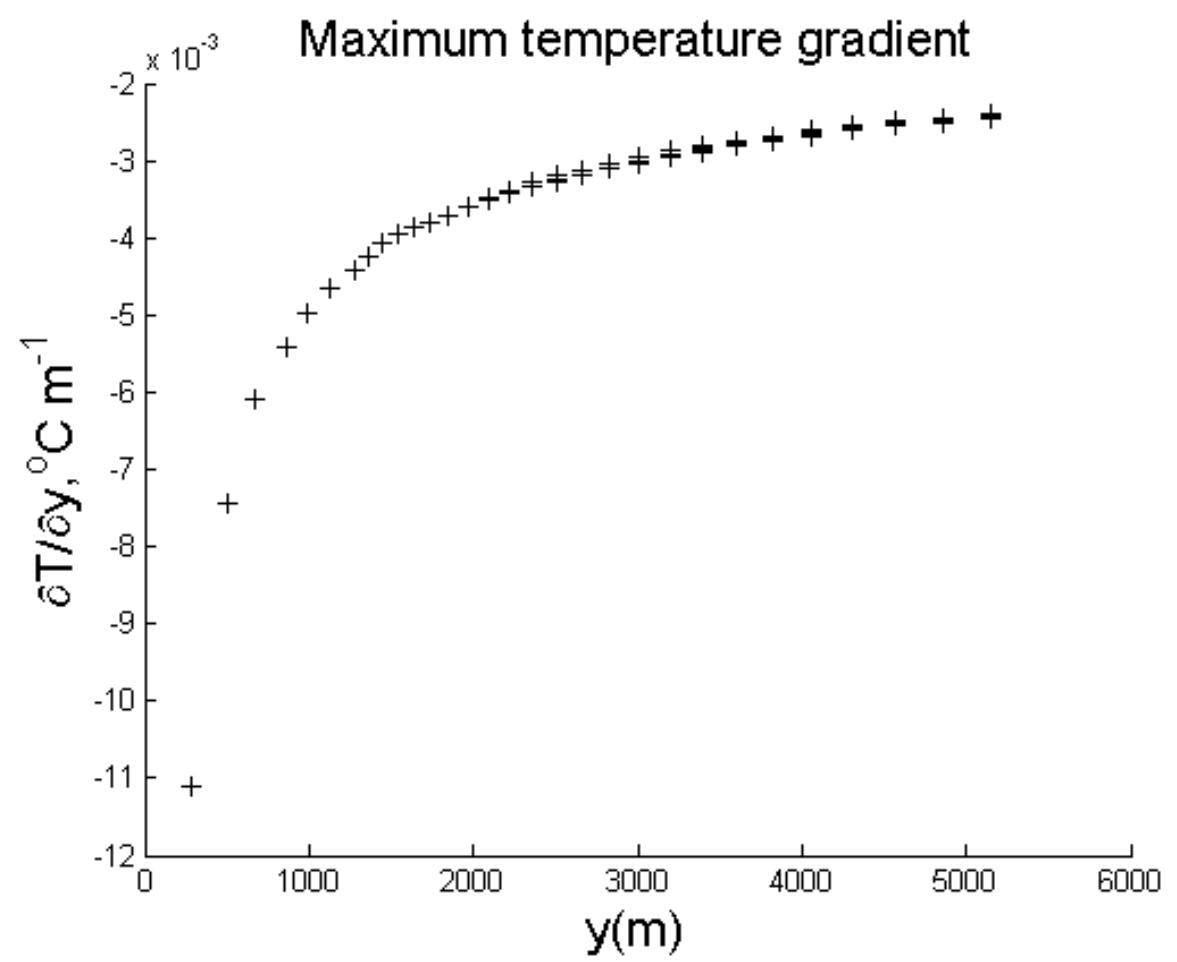

Figure 3.25. Horizontal temperature gradient in the front as a function of the front location (for each of 1 to 150 days ) . 
To explain the change of the frontal intensity, I use the CL model. I consider a front to be a region where the bbl thickness is approximately equal to the local depth. So the temperature distribution in the frontal region can be discussed using the CL model for the bbl. According to this model the bbl is vertically well mixed with vertical isopycnals (Figure 3.26).

Figure 3.26. Schematic representation of the frontal region using CL model. The solid lines are isopycnal. The dashed line is the top of the bbl.

Therefore the temperature is a function of offshore position. Since temperature is continuous through the top of the boundary layer, its distribution in the bbl is determined by the thickness of the bbl and the stratification in the interior. It can be determined as follows:

3.21

$$
\frac{\partial T^{b b l}}{\partial y}=\frac{\partial \delta_{b b l}}{\partial y} \frac{\partial T^{\text {interior }}}{\partial z}
$$

I use the results from the experiment to see how good the CL model works in this case. To do that I compare the frontal temperature gradient to the CL estimate from Equation 3.21. 


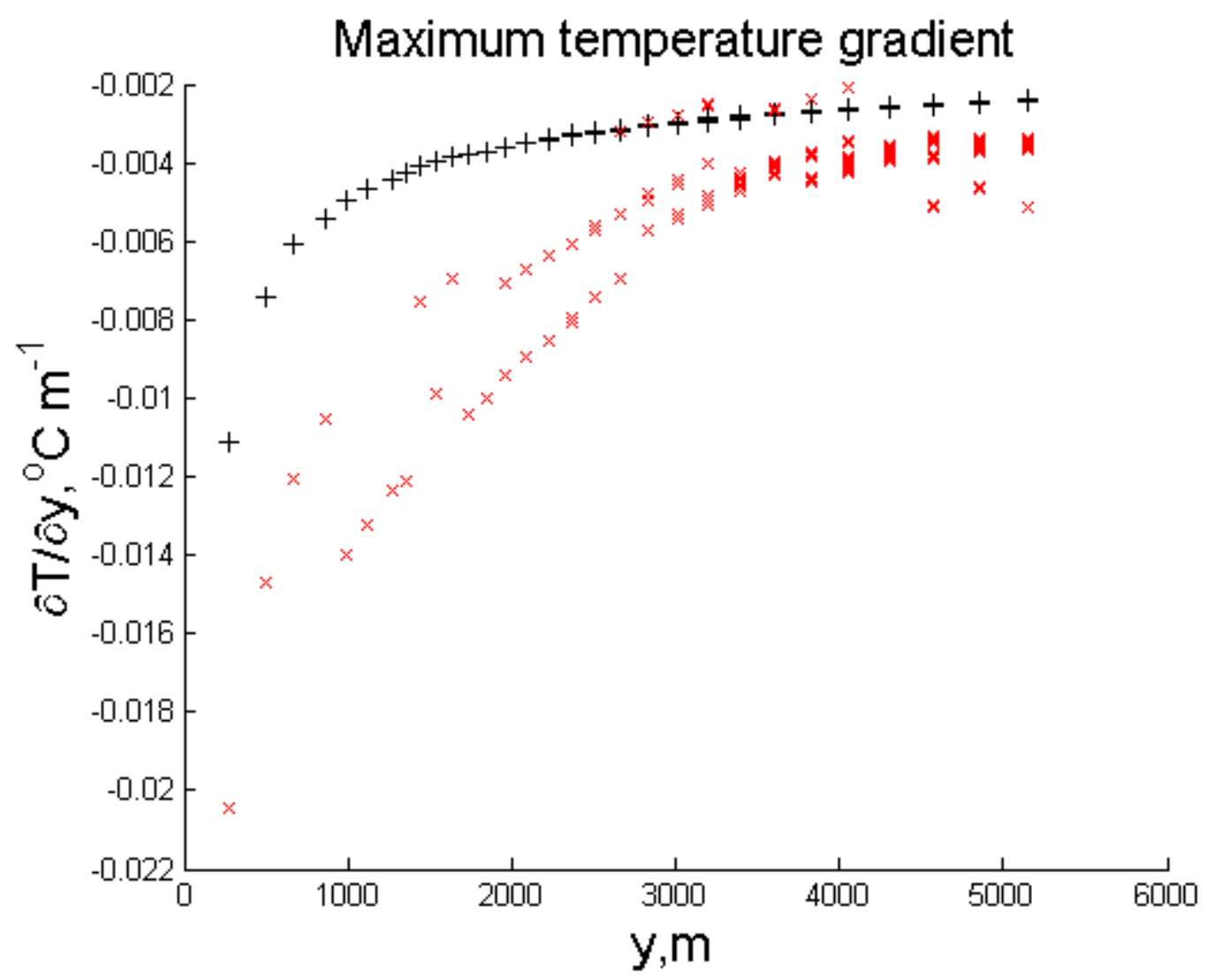

Figure 3.27. The validity of the CL model for predicting the front intensity. The horizontal temperature gradient in the bbl from the pool experiment is in black. The CL model estimate for the gradient (Equation Therefore the temperature is a function of offshore position. Since temperature is continuous through the top of the boundary layer, its distribution in the bbl is determined by the thickness of the bbl and the stratification in the interior. It can be determined as follows:

3.21) is in red.

The comparison shows that the CL model correctly predicts the order of the horizontal temperature gradient (within a factor of two), demonstrating the horizontal scale of the decay of the frontal intensity reasonably well. Data analyses showed that the variation in the interior stratification was insignificant and the variation in the front intensity is mainly caused by the variation in the slope of the bbl top. 


\subsubsection{The alongshore velocity in the vicinity of the front}

As was pointed out above, the alongshore velocity has a vertical shear in the frontal region. As the front moves offshore the size of the barotropic component of the interior alongshore flow decreases and at some location offshore the contribution from the baroclinic component of the alongshore velocity, produced by the frontal horizontal temperature gradient, can exceed the barotropic component contribution. To estimate the value of the baroclinic component I use the scaling analysis. Using the CL model I assume that the frontal horizontal temperature gradient is of the order of the interior stratification offshore of the front (for simplicity I assume it to be equal to the initial stratification) multiplied by the bbl top slope (I consider it to be of the order of the bottom slope):

$$
T_{y}^{\text {front }}=\alpha * T_{z 0}
$$

In the pool experiment $T_{y}^{\text {front }}=1.2 * 10^{-3}{ }^{\circ} \mathrm{C} / \mathrm{m}$. This is an underestimated value, but it can give a scale for a distance offshore, where the barotropic component and baroclinic component have the same magnitude. The scale for the baroclinic component will be 3.22

$$
U^{\text {bar }}=-\frac{\kappa g}{f} * H * T_{y}^{\text {front }}=-\frac{\kappa g}{f} * \alpha^{2} * T_{z 0} * y
$$

As the scale for barotropic component is

3.23

$$
U^{T}=\frac{2 \tau_{0} \exp (-y / L)}{\rho f \delta_{E}}
$$


To get a scale for the distance at which the component are of the same order we need to solve the following equation for $\mathrm{y}$ :

3.24

$$
\frac{2 \tau_{0} \exp (-y / L)}{\rho f \delta_{E}}=-\frac{\kappa g}{f} * \alpha^{2} * T_{z 0} * y
$$

The Equation 3.24 can be solved numerically

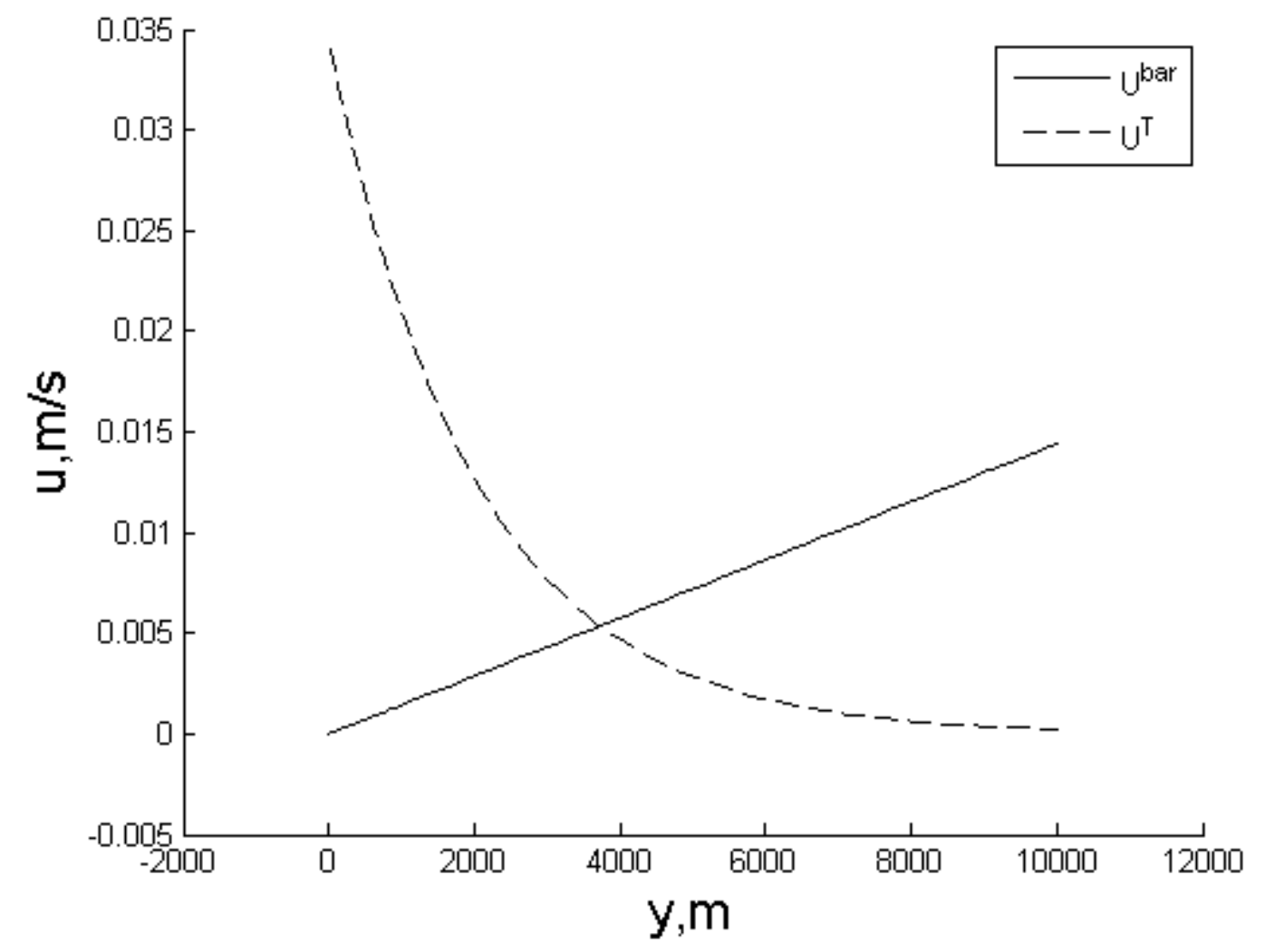

Figure 3.28. Relative importance of the barotropic (dashed) and baroclinic (solid) components. Scaling analysis result using bathymetry and wind stress values from the pool experiment.

So when the front propagates $3730 \mathrm{~m}$ offshore the barotropic and baroclinic components will be of the same order. This estimate is in good agreement with the results from the pool case (Figure 3.19, middle). 


\section{Summary}

I have examined the adjustment of an uniformly stratified wind driven flow over a sloping, frictional bottom using the ROMS numerical model. This work demonstrates that there are two different types of responses to a downwelling favorable wind. I argue that the realization of one or the other response is determined by the magnitude of the applied wind stress, the stratification, the horizontal diffusivity and the Coriolis parameter. In the first case (the bottom boundary layer case), a major feature of the flow was the formation of an unstratified bottom boundary layer. The structure of the layer was observed to have a great impact on the flow in the interior. An offshore temperature gradient in the bbl corresponds to a positive shear in the alongshore velocity. It yields higher velocities in the interior than it would have in the homogenous case. The set of experiments conducted revealed a qualitative dependence of the wind response on the initial stratification, the interior diffusivity and the bottom slope. It was shown that the bbl thickness is proportional to the stratification, the slope and magnitude of the applied wind stress and inversely proportional to the background diffusivity. This is in qualitative agreement with Pedlosky (2007). There it was also shown that a lower background (interior) diffusivity and a steeper slope correspond to a thinner (vertically) and narrower (horizontally) bbl.

The major difference in the second case is that an unstratified pool region is formed near the shore, which is separated by a front-like structure from the uniformly stratified region offshore. A bbl-like layer is present on the offshore side of the pool region and the pool region expands with time. Using an idealized model, I demonstrated that the expansion rate is inversely proportional to the local depth and wind stress and diminishes as the front moves offshore. The front region has an enhanced offshore temperature gradient corresponding to a positive vertical 
shear in alongshore velocity. The intensity of this front was shown to be determined mainly by the slope of the bbl top.

In this study, I used a highly simplified model of the ocean. One of the simplifications that are made is the two-dimensional configuration, which is far from being realistic. A study, that considered the three-dimensional problem, is highly desirable. Also it would be interesting to consider a time dependent wind response, which is also more realistic than the model presented here. 


\section{Appendix}

\section{Weakly stratified pool formation criterion.}

In this section, we use scaling arguments to derive a pool formation criterion. I consider the region immediately next to the coastal wall. The warm water from the surface is advected downward in the hydrostatic layer with a horizontal extent of the order of the Rossby deformation radius. In the beginning, there is no mechanism to balance the temperature anomaly, brought by vertical advection in this layer. Therefore, the local temperature throughout the water column rises yielding Equation 5.1. This can be observed as the isopycnals are pushed down along the wall.

5.1

$$
\frac{\partial T}{\partial t}+w \frac{\partial T}{\partial z}=0
$$

This process generates horizontal variation in the temperature field. This variation gives rise to diffusive processes. As time progresses, the vertical advection of temperature decreases owing to the reduction in the vertical temperature gradient and eventually is balanced by the horizontal diffusion yielding Equation 5.2:

\section{2}

$$
w \frac{\partial T}{\partial z}=\frac{\partial}{\partial y}\left(K_{H} \frac{\partial T}{\partial y}\right)
$$

Here I argue that the criterion for the well-mixed pool formation is that the surface isopycnal intersects the bottom. In other words, the surface water was advected all the way to the bottom without losing much of its temperature to diffusion. So the pool will not be formed if the 
advective time scale is much larger than the diffusive time scale, or $\frac{\tau_{a d v}}{\tau_{d i f f}} \gg 1$. The value of this ratio can be evaluated as

5.3

$$
\begin{gathered}
\tau_{a d v}=\frac{D}{W}=\frac{D \rho f \delta}{\tau_{0}} \\
\tau_{\text {diff }}=\frac{\delta^{2}}{K_{H}}
\end{gathered}
$$

Where $\delta=\frac{N D}{f}=\frac{\sqrt{\kappa g T_{z}}}{f} D$ is the thickness of the side wall boundary layer; $N$ is the initial buoyancy frequency; $\kappa$ is the thermal expansion coefficient; $T_{z}$ is the initial vertical temperature gradient; $\mathrm{W}$ is the scale for the vertical velocity in the hydrostatic layer (obtained using continuity equation and the Ekman transport), $D$ is the depth of the coastal wall.

\section{4}

$$
\frac{\tau_{a d v}}{\tau_{d i f f}}=\frac{D \rho f \delta}{\tau_{0}}\left(\frac{\delta^{2}}{K_{H}}\right)^{-1}=\frac{K_{H} \rho f^{2}}{\tau_{0} \sqrt{\kappa g T_{z}}}
$$

This relationship demonstrates that the higher the wind and the stratification the more likely the pool formation. Using Equation 5.4 the criterion $\frac{\tau_{a d v}}{\tau_{d i f f}} \gg 1$ can be rewritten as a condition on the wind stress amplitude:

5.5

$$
\tau_{0} \ll \frac{K_{H} \rho_{0} f^{2}}{\sqrt{\kappa g T_{z}}}
$$

So if the wind amplitude is much less than $\tau_{0}$ then the pool region will not form. In the case when the wind amplitude is of the order of $\tau_{0}$ or larger, the pool region will be formed. This criterion is in good agreement with the experimental results. For example, in the pool experiment 
the value of the ratio is 1 and in the bc experiment it is 63.5. The rest of the conducted experiments also demonstrate this agreement. 


\section{Bibliography}

Chapman,D.C. and S.J. Lentz . (1997). Adjustment of Stratified Flow over a Sloping Bottom. Journal of Physical Oceanography, 340-356.

Allen, J.S., Newberger, P.A. (1996). Downwelling Circulation on the Oregon Continental Shelf. Part I:Response to idealized Forcing. Journal of Physical Oceanography, 2011-2035.

Allen,J.S. and P.A. Newberger. (1996). Downwelling circulation on the Oregon Continental Shelf: Part 1, Responce to Idealized Forcing. J.Phys.Ocean. , 2011-2035.

Austin, J. a. (2002). The Inner Shelf Responce to Wind-Driven Upwelling and Downwelling. J. Phys. Ocean, 2171-2193.

Barcelon, V. and J. Pedlosky. (1967). Linear thory of rotating stratified fluid motions. J. Fluid Mech., 1-16.

Ekman, V. (1905). On the Influenceof the Earth's Rotation on Ocean-Currents. Arkiv for Mathematik; Astronomi och Fysik, Band 2, No. 11.

Ezer, T. A. (2002). Developments in terrain-following ocean models: intercomparisons of numerical aspects. Ocean Modelling , 249-267.

Garrett, C., P.MacCready, and P.Rhines. (1993). Boundary mixing and arrested Ekman layers: Rotating stratified flow near a sloping boundary. Annu. REv. Fluid Mech. , 291-323.

Lopez-Mariscal, M. and A. J. Clarke. (1993). On the influence of wind-stress curl on lowfrequency shelf water flow. J. Phys. Ocean , 2717-2727.

MacCready, P. and P.B. Rhines . (1993). Slippery bottom boundary layers on a slope. J.Phys. Ocean. , 5-22.

Pedlosky, J. (1987). Geophysical Fluid Dynamics. Springer-Verlag. 
Pedlosky, J. (2007). The coastal bottom boundary layer: a note on the model of Chapman and Lentz. J.Phys. Oceanogr. , 2776-2784.

Shchepetkin, A., F. and J.C. McWilliams. (2005). The regional oceanic modeling system (ROMS): a split-explicit, free-surface, topography-following-coordinate oceanic model. Ocean Modelling , 347-404. 